\title{
Bactericidal Effects of Natural Nanotopography of Dragonfly Wing on Escherichia coli
}

Chaturanga D. Bandara ${ }_{\dot{\dagger}+}^{+}$, Sanjleena Singh ${ }^{\S}$, Isaac O. Afara ${ }^{i}$, Annalena Wolff, Tuquabo

Tesfamichael ${ }^{*}$, Kostya (Ken) Ostrikov $v^{\ddagger \xi}$, Adekunle Oloyede

${ }^{\dagger}$ School of Chemistry, Physics and Mechanical Engineering, Science and Engineering Faculty, Queensland University of Technology (QUT), Brisbane, Queensland 4001, Australia

Institute for Health and Biomedical Innovation (IHBI), Queensland University of Technology (QUT), Kelvin Grove, Queensland 4059, Australia

${ }^{\S}$ Institute for Future Environments, Queensland University of Technology (QUT), Brisbane, Queensland 4001, Australia

${ }^{i}$ Research and Innovation Centre, Elizade University, 1 Wuraola Ade.Ojo Avenue, P.M.B 002, Ilara-Mokin, Ondo State, Nigeria

KEYWORDS: bactericidal, surface science, topography, dragonfly and helium ion microscopy 


\begin{abstract}
Nano-textured surfaces (NTS) are critical to organisms as self-adaptation and survival tools. These NTS have been actively mimicked in the process of developing bactericidal surfaces for diverse biomedical and hygiene applications. To design and fabricate bactericidal topographies effectively for various applications, understanding the bactericidal mechanism of NTS in nature is essential. The current mechanistic explanations on natural bactericidal activity of nanopillars have not utilized recent advances in microscopy to study the natural interaction. This research reveals the natural bactericidal interaction between E.coli and a dragonfly wing's (Orthetrum villosovittatum) NTS using advanced microscopy techniques and propose a model. Contrary to the existing mechanistic models, this experimental approach demonstrated that the NTS of Orthetrum villosovittatum dragonfly wings has two prominent nanopillar populations and the resolved interface shows membrane damage occurred without direct contact of the bacterial cell membrane with the nanopillars. We propose that the bacterial membrane damage is initiated by a combination of strong adhesion between nanopillars and bacterium EPS layer as well as shear force when immobilised bacterium attempt to move on the NTS. These findings could help guide the design of novel bio-mimetic nanomaterials by maximising the synergies between both biochemical and mechanical bactericidal effects.
\end{abstract}

\title{
INTRODUCTION
}

Various organisms living in nature use nano-textured surfaces (NTS) to adapt, interact and survive in their environments. Adhesion, ${ }^{1-3}$ anti-reflection, ${ }^{4-6}$ bactericidal activity, ${ }^{7-11}$ superhydrophobicity, ${ }^{12-16}$ and dew condensation ${ }^{17}$ are some of the properties exhibited due to their surface chemistry and topographical structure at the micro and nano-scale. For example, the anti- 
biofouling properties of NTS of the lotus leaf ${ }^{18}$ and shark skin ${ }^{19}$ are a consequence of their surface topography while wings of cicada ${ }^{9-11}$ and dragonfly $^{7-8}$ possess bactericidal (See Table S1) and anti-reflective ${ }^{4-5}$ properties to protect them from their natural predators. Dragonflies are renowned for their striking aerodynamic performance and their wings have been extensively studied at the macro-scale. ${ }^{20-22}$ Maintaining the aerodynamic performance is of vital importance for the dragonfly's survival. This requires a clean and light wing span which can be achieved if dust particles are easily washed off (superhydrophobicity) and bacterial growth is avoided in moist environments (bactericidal properties). Both properties of the wings are achieved by micro and nano scale surface topographies ${ }^{7-8}$ which are built by fatty acids and long chain $n$-alkanes. ${ }^{23}$ Inspired by this natural phenomenon, various artificial NTS including nanocones, nanofibers, and nanopillars are fabricated from different materials to achieve bactericidal properties. ${ }^{24-26}$ (See Table S2) Most of the fabricated NTS deviate from the natural NTS, both in chemistry and architecture, hence bactericidal efficiencies of artificial bio-inspired surfaces are different to natural bactericidal NTS. ${ }^{7,25,27}$ Therefore optimisation of bactericidal efficiency of the bioinspired fabrications has proven challenging and requires concise understanding of both bactericidal mechanism and topography of natural NTS, prior to fabricate nanotextures onto various applications.

Three prevailing explanations for bacterial death on cicada wing nanopillars proved to be mechanical in nature and include; 1) direct penetration of bacterial membrane by the NTS, ${ }^{9}$ 2) stretching and rupture of the membrane in between NTS, ${ }^{28-30}$ and 3) membrane deformation due to its adhesion with the NTS. ${ }^{31}$ These mechanistic explanations on natural bactericidal activity of nanopillars are based on assumptions that nanopillars make direct contact with the bacterial membrane and nanopillars are strong enough to damage the bacterial membrane. In the literature 
moduli of cicada wing membrane are reported to be $3.7 \mathrm{GPa}{ }^{32}$ The conventional understanding is that insect wings are made of chitin and cuticular waxes. ${ }^{9,33-36}$ Therefore the nanopillars on cicada wing are assumed to be strong enough to damage the bacterial membrane. ${ }^{9} \mathrm{Li}$ et al ${ }^{30,37}$ and Xue et $\mathrm{al}^{29}$ investigated the bacterial membrane rupture within the framework of stretching theory. Li et al show that the physical origin of the bacterial adhesion on a nanopillar surface is determined by the balance between adhesion energy and the deformation energy of the cell membrane. ${ }^{37}$ The large surface roughness due to nanopillars increase the contact surface area, while the small radius of nanopillars increase the membrane deformation energy. ${ }^{37}$ Therefore the thermodynamic model show the importance of architecture and the density of nanopillar arrangement for efficient bactericidal activity. ${ }^{30}$ However, in the mathematical model developed by Xue et al suggested that sufficient energy that would lead to full adhesion and membrane rupture cannot be provided only by the physical interactions between bacterial membrane and the nanopillar structures, but also it requires the gravitational force. ${ }^{29}$ These studies suggest that the changes in the architecture and density of nanopillars affect the efficiency of the bactericidal activity.

Bacteria show different strategies to interact and adhere on to abiotic surfaces using nonspecific adhesins; fimbrial, non-fimbrial, and discrete polysaccharides. ${ }^{38-40}$ Extra-cellular polymeric substance (EPS) is a non-specific adhesins used by E.coli as molecular glue to attach on to surface. ${ }^{38,41}$ It is to be noted that the current mechanistic explanations of bacterial death on NTS do not consider the adaptability and variability shown by bacteria, ${ }^{42}$ the presence of protective peptidoglycan layer (PG) in bacteria and secretion of EPS by bacteria during interaction with NTS. Recent advances in microscopy have not been utilised to confirm those mechanistic explanations and some contrasting studies have shown that bacteria can self-repair 
their cell wall and retain their integrity, viability and reproduction even after repeated punctures by a sharp AFM tips. ${ }^{43-44}$

In this research, we reproduced the natural attachment of bacteria on nanopillars of dragonfly wing, to understand the bactericidal mechanism. Advanced microscopy techniques were employed to investigate the nanotopography, and mechanical properties of the wing's nanopillars and observed successive events which occur at the interface between a bacterial cell and the nanopillars. Helium Ion Microscopy (HIM), Atomic Force Microscopy (AFM), Transmission Electron Microscopy (TEM), and FIB/SEM Microscopy are the techniques used in this study. The results indicated that the wing topography comprises of two prominent populations of nanopillars in contrast to the single height structure commonly mimicked in biomaterial and experimental models. Bacterium is attached onto nanopillars via secreted EPS and its membrane has no direct contact with the nanopillars as the EPS is filled between the nanopillars and the bacterial membrane. The large surface area due to the nanopillar arrangement causes strong adhesion of the bacterium onto surface through the EPS. Movement of bacterium on the wing surface cause bending of the taller nanopillars. During this interaction, bacterial membrane damage was identified as a separation of the inner-cell membrane from the outer-cell membrane. At the same time, separation of nanopillars layer from wing base just under the bacterium is also observed. Furthermore we observed that once the membrane is damaged cell cytoplasm leaks onto the wing which then flows under the nanopillars layer. It is reported in the literature that strong adhesion and shear stress are lethal to bacteria. ${ }^{45-47}$ Previous studies also showed cylindrical shaped nanopillars similar to that found on dragonfly wing are capable of applying larger shear force on a cell membrane but with a shallow indentation compared to a cone or pyramidal shaped nanopillars. ${ }^{48}$ From the above analysis, we propose a bactericidal mechanism 
of dragonfly wing nanopillars are due to a combined effect of strong adhesive force between bacterium-nanopillars and shear force when immobilised bacterium attempts to move on the nanopillar surface.

\section{RESULTS AND DISCUSSION}

The topography of dragonfly wing is characterized using HIM, TEM and AFM and is presented in Figure 1. The native nanotextured surface of the delicate, insulating biological material could be visualized using HIM associated with a floodgun to compensate the accumulated ion charge which interferes during imaging. The helium ion micrograph (Figure 1a) shows a dense array of closely but randomly-arranged two prominent populations of nanopillar architecture. These nanopillars are cylindrical in shape with about the same diameter throughout their length, terminating with a slight curvature at the top. These pillars arise from a horizontal network of ridges (arrow heads in Figure 1a) on the base of the wing. Some voids (outlined in dotted lines) can also be seen between the network of ridges. (Figure 1a) 

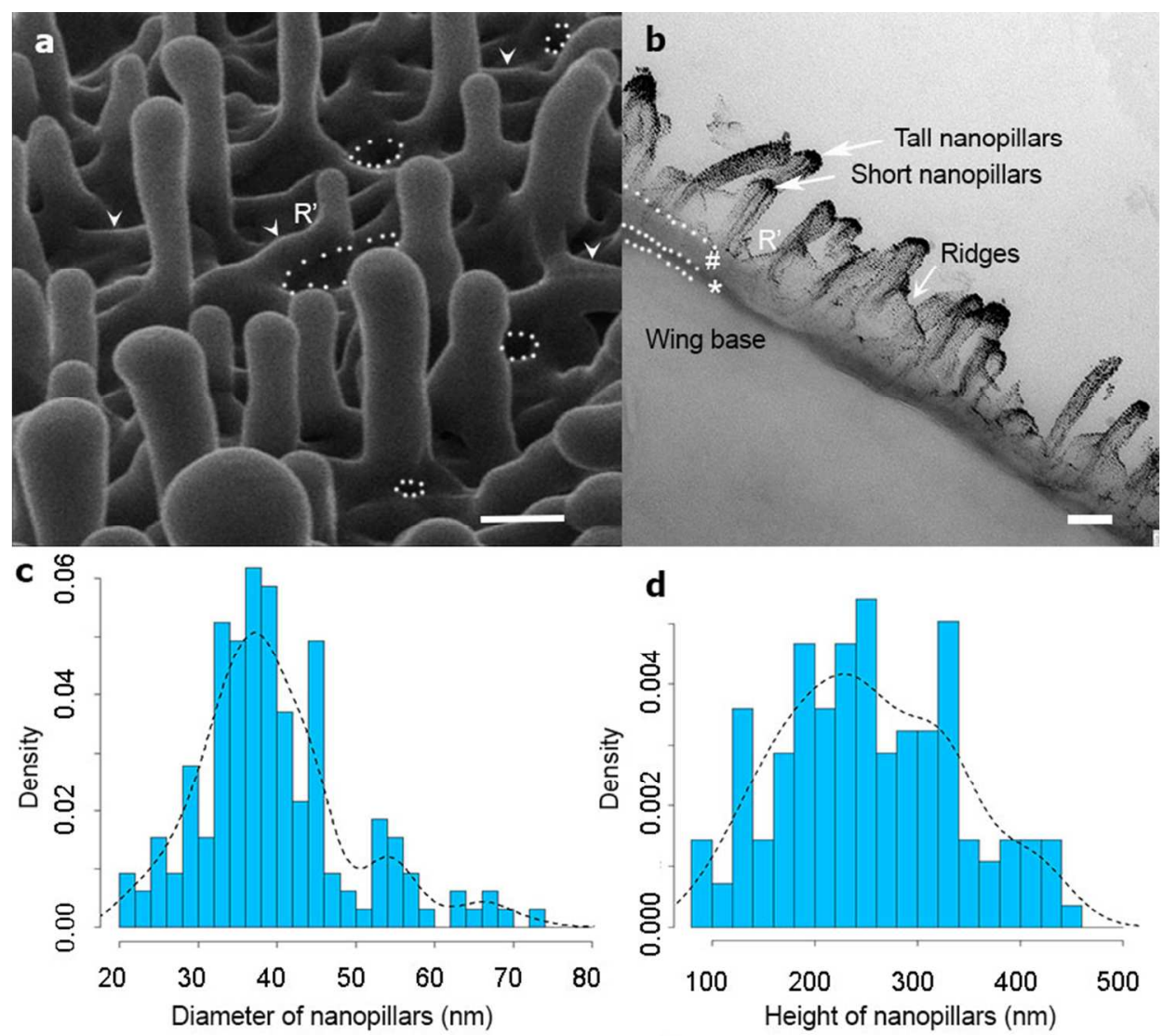

e
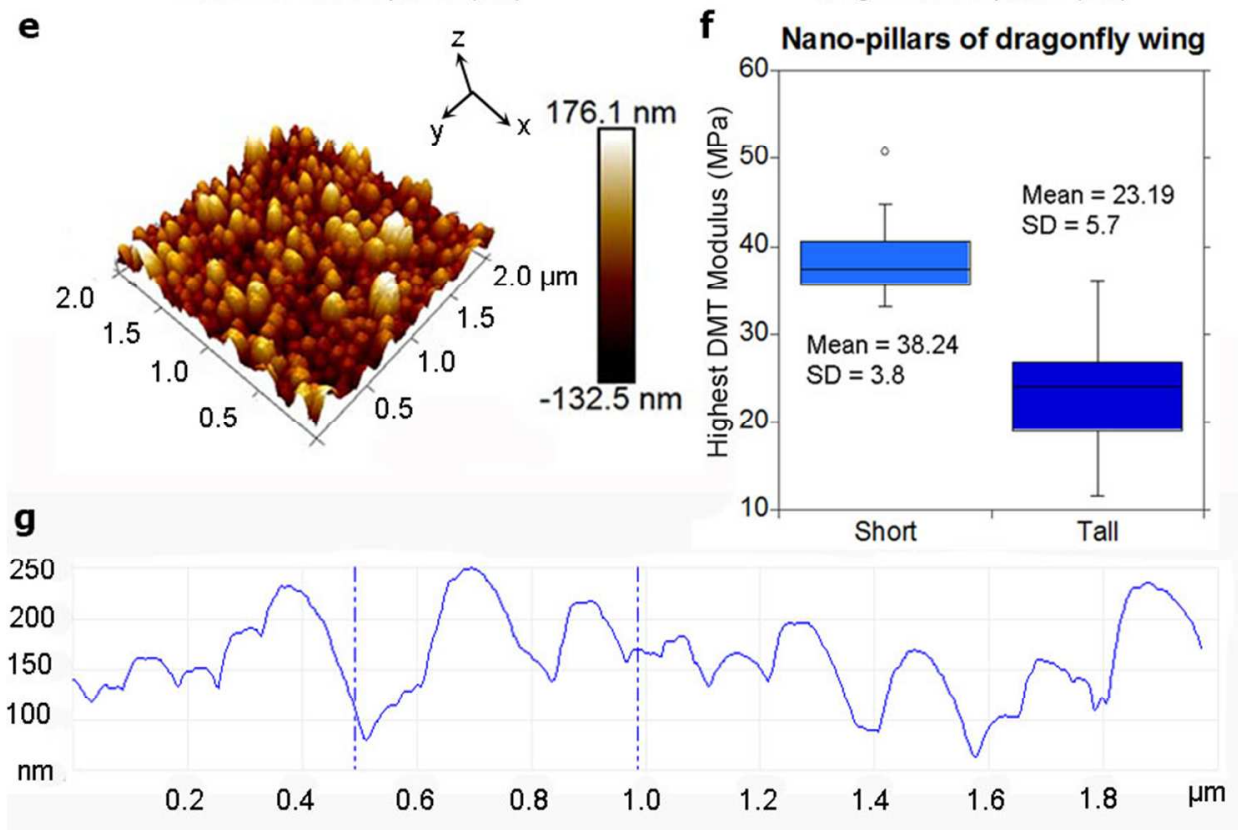

Figure 1: Hierarchical two-prominent distributions in nanopillar topography of a dragonfly wing. a) Helium Ion micrograph showing the native nanopillars arrangement on the dragonfly wing. Nanopillars arise from the horizontal ridges (arrow heads) at the base. The voids in the 
horizontal network are outlined by dotted lines. The scale bar corresponds to $200 \mathrm{~nm}$. b) TEM micrograph showing a cross-sectional view of the wing and its nanotopography. Tall and short nanopillar arrangement can be observed. Wing base is more dominant and nanopillar layer is attached to wing base via a tiny sandwiched layer (*). The scale bar corresponds to $200 \mathrm{~nm}$. (R' in HIM and TEM show a nanopillar arise from a ridge). c) Histogram showing nanopillar diameters and their distribution (dotted line) obtained from TEM image analysis. d) Histogram showing nanopillar heights and their distribution (dotted line) obtained from TEM image analysis. e) Atomic force micrograph of the wing's surface topography showing that the tall nanopillars are randomly distributed among the short nanopillars. f) Average reduced modulus of shorter and taller nanopillars. g) Cross-sectional profile of AFM topography showing the twodifferent height arrangement in nanopillar topography.

TEM cross-sectional view of the specimen (Figure 1b) shows layered organisation of the wing structures with nanopillars arise from the horizontal network of ridges (labelled as \#). For comparison of the nanopillars arise from these ridges are labelled as R' in both TEM and HIM micrographs. Collectively, nanopillars and ridges are identified as the nanopillar layer (NPL). This NPL is attached to wing base (WB) via a thin layer (labelled as *), which is sandwiched between NPL and WB. The TEM Cross-sectional view of the dragonfly wing also shows the variation in pillar heights (Figure 1b) as observed in HIM. The nanopillar diameter (Figure 1c) and height (Figure 1d) distributions are evaluated from the cross-sectional TEM micrographs of the wing. The shorter nanopillars are more abundant than the taller ones (Figure 1d). The average calculated height of the taller and shorter nanopillars and diameter distributions from TEM analysis are given in Table 1 and Figure 1c respectively. The distribution of nanopillar heights was normal according to Anderson-Darling normality test with a p-value of 0.1931 while that for 
diameter was not normal with p-value of $2.194 \times 10^{-6}$. However, according to Galtung's AJUS classification system for distributions, nanopillar height and diameter populations are found to be S type distributions, which represents bimodal or multimodal peaks in their distributions ${ }^{49}$ and are found in several natural populations. ${ }^{4,50-51}$ AFM is one of the most commonly used methods to compare surface topographies. However, when AFM is used to characterize high-aspect ratio topography, it requires special cantilever. We have used a cantilever which can measure the mechanical properties with a reasonable topography mapping. As this tip is not designed for characterizing the entire high aspect ratio nanopillars on the wing, the cantilever tip do not travel the entire height scale of the nanopillars, and do not provide micrograph as in HIM and TEM. More details are given in the supplementary section. As such, we have used the TEM to measure the height distribution of nanopillars on the wing. Given that the sampling thickness is $100 \mathrm{~nm}$, and thickness of nanopillar is about half of that size, statistically there is less chance to include a slice of single pillar being analysed but high chance of analyzing the entire pillar captured in the cross-section. 
Table 1: Properties of dragonfly wing nanopillars and bacterium

\begin{tabular}{|l|c|c|c|c|}
\hline \multirow{2}{*}{} & \multicolumn{2}{|c|}{ Wing } & \multicolumn{2}{c|}{ Bacterium } \\
\cline { 2 - 5 } & Short pillar & Tall pillar & Cell Membrane & PG $^{1}$ layer \\
\hline Height (nm) & $189 \pm 67$ & $311 \pm 52$ & - & - \\
\hline Reduced modulus & $38 \pm 3.8$ & $23 \pm 5.7$ & $(50-150)^{52-54}$ & $(45-60)^{52,55}$ \\
$(\mathrm{MPa})$ & & & & \\
\hline Ra (RMS) (nm) & \multicolumn{2}{|c|}{$32(41)$} & N/A & N/A \\
\hline Pillar diameter (nm) & $37 \pm 6$ & $57 \pm 8$ & N/A & N/A \\
\hline
\end{tabular}

AFM measurements were performed using Peak Force tapping ${ }^{\mathrm{TM}}$ (PFT) mode with Scan Assist $^{\mathrm{TM}}$ enable imaging of the wing surface with minimal contact force to minimise damage to the surface and avoid tip artefacts. ${ }^{56}$ The data shows the presence of a two prominent populations in height distribution of the nanopillars (Figure 1e) which is in good agreement with the HIM and TEM results. Mechanical property mapping was done with the PeakForce QNM ${ }^{\circledR}$ (quantitative nanomechanical mapping) of AFM. The obtained force curves for each individual tap by PFT AFM were converted to force versus separation plot for further analysis using NanoScope Analysis software. These force-separation curves are analogous to the loadindentation curves used in nanoindentation. To obtain modulus, the retract curve was fitted using the Derjaguin-Mullet-Toporov (DMT) model. ${ }^{57}$ The measurements also show that the shorter nanopillars are more rigid, with an average reduced modulus of $38 \pm 3.8 \mathrm{MPa}$ than the taller nanopillars which have an average modulus of $23 \pm 5.7 \mathrm{MPa}$ (see Figure $1 \mathrm{f}$ and Table 1). For the 
statistics, 30 individual measurements were taken for each set of tall and short nanopillars. These values of nanopillars are much less than the modulus of the insect wing membranes previously reported. In literature modulus of different dragonfly wing membranes evaluated from tensile and nanoindentation methods are reported in the range of $1 \mathrm{GPa}-2.85 \mathrm{GPa}^{35,58}$ Dragonfly wing membrane modulus reported in literature are much less to $\alpha$-keratin, (4 GPa) and $\beta$-keratin (8$10 \mathrm{GPa}$ ) the components which make the cuticle structure of insects, but higher than amorphous protein polymers $(1.2 \mathrm{MPa}){ }^{59-60}$ The values in literature were reporting the mechanical properties of the entire wing membrane but not the functional nanopillars. ${ }^{32,58,60}$ There are no attempts have been found in literature on attempts to measure the mechanical properties of the nanopillars on the dragonfly wing to our best understanding. A recent study connecting chemistry and topography of nanopillars on dragonfly wing has reported that they are made of fatty acids, dominating with hexadecanoic acid $n$-alkanes and even numbered carbon chains ranging from $\mathrm{C}_{14}-\mathrm{C}_{30},{ }^{23}$ which is different to the conventionally accepted understanding that insect cuticles are network of chitin. ${ }^{34-36}$ Therefore the modulus of nanopillars determined by AFM QNM are expected to be much less than previously reported in literature, and this is the first time of reporting such mechanical properties of the nanopillars on insect wings. Due to the two prominent nanopillar populations of the dragonfly wing, the average roughness $(\mathrm{Ra})$ and root mean square roughness (RMS) are found to be large (32 nm and $41 \mathrm{~nm}$, respectively). From the $2 \mu \mathrm{m} \times 2 \mu \mathrm{m}$ scanned AFM micrograph, the surface area of the nanopillars was determined to be $7.06 \mu \mathrm{m}^{2}$ and this is higher by about $77 \%$ compared to a flat surface area $(4 \mu \mathrm{m})$.

The topographical details of dragonfly wing tested in this study using HIM, AFM and TEM (Figure 1) are similar to each other but they differ from the nanotopography obtained using SEM (Figure S1). These SEM micrographs are similar to that of previous studies ${ }^{7-8}$ and did not reveal 
the two prominent height populations of nanopillars. The incident electron beam was found to alter the original nanopillar structure by consolidating adjacent nanopillars during SEM imaging. HIM, AFM and TEM overcome the limitations encountered in SEM and showed that the native dragonfly wing nanopillar topography is more complex than previously reported. ${ }^{7-8}$

Bactericidal efficiency of the dragonfly wing against Escherichia coli was assessed over a four hour time period (Figure 2). The state of bacteria after attachment to the wing is compared using SEM micrography on control glass surface (Figure 2a) and tested nanopillar surface (Figure $2 \mathrm{~b}$ ). The bacteria attached on the glass surface appears to have the native round shape which is a typical identification of live bacteria where as the majority of the bacteria attached on the wing surface are flattened, an indication of dead cells. The nanopillars arround the flatened bacterial cells apper to be bent towards the cells at this magnification. The flattened bacteria on nanopillars is due to the leak of cytoplasm after their membrane damage has occurred and it has good agreement with the previously reported data. ${ }^{7,9,} 11$ Insets in Figure 2a-b show close up images of the live and dead bacterial morphologies, respectively. 


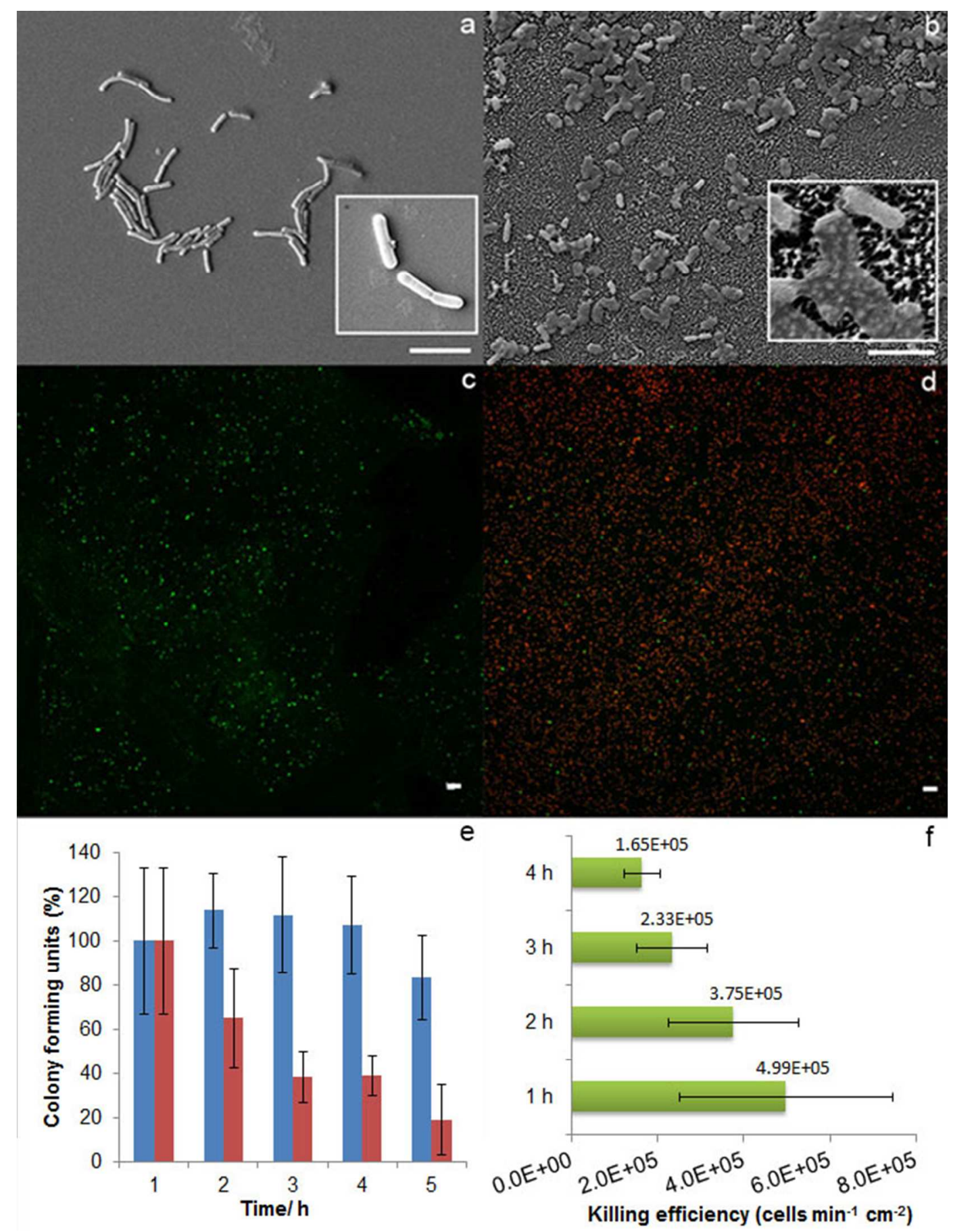

Figure 2: Comparisons of the dragonfly wing's bactericidal properties with a flat control glass surface a) SEM micrograph showing morphology of E.coli on a flat control glass surface. Their round shapes show the cell's survival on the control substrate b) SEM micrograph showing morphology of E.coli on the dragonfly wing. The majority of cells is flattened indicating cell 
death and does not exhibit a round shape. c) Confocal images showing the bacterial cells after 15 minutes on the control glass substrate. The green stain shows the membrane intact cells on the control substrate. d) Confocal image showing the bacterial cells after 15 minutes on the dragonfly wing. The red stain indicates membrane damage, indicating major cell death on the natural nanopillars. Only a few bacteria survive on this substrate which is shown in the minor green stain signal. e) Statistics of percentage of cells survived at each time interval on both the flat control surface (blue) and the dragonfly wing (red). f) Dragonfly wing's killing efficiency over $4 \mathrm{~h}$ of time.

The live/dead state of bacteria on the control glass surface and dragonfly wing surface (Figure 2d) is discriminated using confocal microscopy. The red color indicates the membrane damaged bacteria and green color indicates membrane intact bacteria. On the glass surface, all cells are stained green (Figure 2c), indicating that the cells are alive. In contrast, on the dragonfly wing most of the bacteria are stained red, with a few stained green (Figure 2d). This indicates that the majority of the bacteria on the dragonfly wing are dead. This observation is in good agreement with the SEM images in Figure 2a-b.

Live bacterial numbers on the dragonfly wing are highly reduced in comparison to the number of bacteria survived on control glass substrate as shown in Figure 2e. This indicates that the bacterial cell death with time is much higher on the dragonfly wing compared to the death occurred on the control surface. The calculated bactericidal efficiency of dragonfly wing against Escherichia coli decreases with time as shown in Figure $2 \mathrm{f}$. After $3 \mathrm{~h}$ the efficiency is $2.33 \times 10^{5} \pm 5.83 \times 10^{4}$ cells $\mathrm{min}^{-1} \mathrm{~cm}^{-2}$ and this value is slightly less compared to the reported bactericidal efficiencies of dragonfly wing ${ }^{7}$ for Staphylococcus aureus $\left(4.5 \times 10^{5}\right.$ cells $\left.\mathrm{min}^{-1} \mathrm{~cm}^{-2}\right)$ and Pseudomonas aeruginosa $\left(4.3 \times 10^{5}\right.$ cells $\left.\mathrm{min}^{-1} \mathrm{~cm}^{-2}\right)$ (Table S1). However, it is noticable that 
the bactericidal efficiency decreases from $4.99 \times 10^{5}$ to $1.65 \times 10^{5}$ cells $\mathrm{min}^{-1} \mathrm{~cm}^{-2}$ with time. To determine whether the killing efficiencies at each hour is significantly different to each other, Kruskal-Wallis $\mathrm{H}$ test were performed. The obtained p-value of 0.536 suggests that the killing efficiencies reported over $4 \mathrm{~h}$ time period are not significantly different to each other. The reduction of bactericidal efficiency over the time period may be due to the combined effects of the following reasons. With the time, number of live bacteria in the sample is decreasing and as bacteria attaches to the surface and dies, therefore the bactericidal surface area is slao decreasing. In the mile and mistra method, live CFU were counted by culturing the diluted colonies at each time interval. This procedure includes preparation of dilution series for both control and test sample, and the colonies in the culture were counted after an overnight incubation and plating the dialutions. Eventhough the colonies for each aliquot between 3-30 were counted, two independent populations from test sample and control samples are considered to calculate the number of dead cells and killing efficiency. Therefore, the added variance from two individual samples is higher. With multiple calculation steps adding variance from two individual samples considered, the calculated error in killing efficiency is quite high.

Currently, we have done the study selecting a single area of the wing considering the difficulties related to macro, micro and nanoscale roughness of wing experienced during various measurements. As the wing is wavy, hydrophobic and holds roughness at multiple scales, it is difficult to repeat all the AFM, HIM, TEM with bacteria culture tests at different locations of the wing looking for various distributions of tall/short nanopillars. This multiscale roughness limits the characterization of the possible various distributions of tall/short nanopillars within the same wing. However, the microscopic analysis confirmed that the entire wing contains quite similar topography. Therefore, we have tested and reported the killing efficiency of a particular area of 
the wing where the wing appeared to be non-wavy visually at a macro scale. We haven't identified the areas of the wing, where significant difference in distribution of both the tall/short nanopillars and we haven't done any calculations that relate the killing efficiency with respect to different tall/short distributions of the nanopillars at this time. This may require adaptation of different species of dragonfly wing to find the surfaces with such significant differences in the tall/short nanopillar distributions.

Figure 3 shows HIM micrographs of Escherichia coli attached on to the dragonfly wing via the secreted EPS to attach them on to the surface. At this magnification surrounding nanopillars appeared to be bent towards the bacterium (Figure 3a). The inset show a low magnification SEM of Escherichia coli attached to dragonfly wing and the surrounding nanopillars are bend towards the bacterium. This is similar to reported observation in previous studies using SEM. ${ }^{8-9,} 11$ However at higher magnification with better spatial resolution the interaction is clearly resolved with greater details; bacteria use EPS and finger-like extensions (Figure 3b-e) to attach onto surrounding nanopillars. These finger-like extensions are about the same in diameter to the nanopillars on dragonfly wing. Here at higher magnification, it is clearly resolved that surrounding nanopillars do not bend towards the bacterium, but the EPS and finger-like extensions from bacteria were appeared as surrounding nanopillars bend towards the bacterium at low magnified images. 


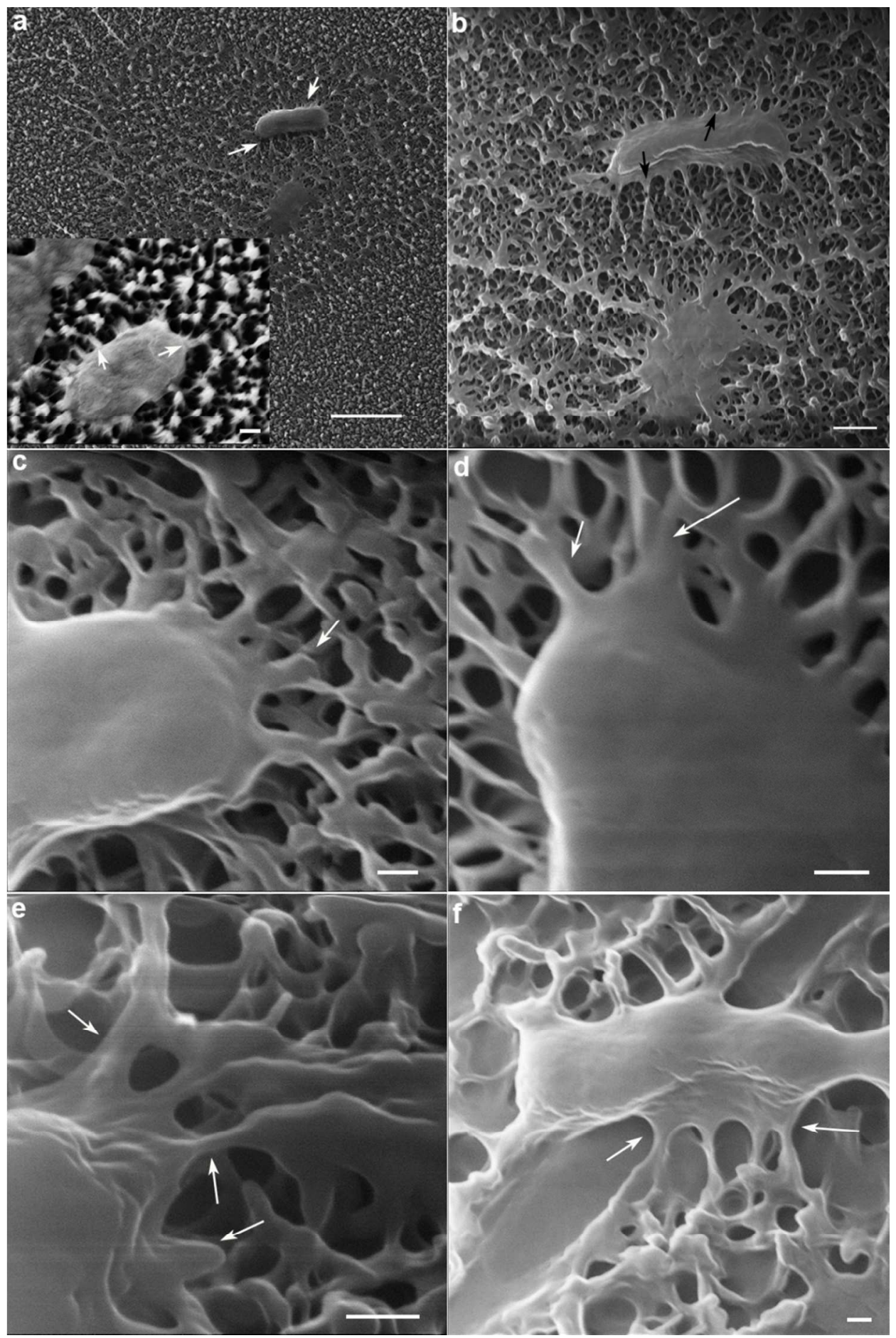

Figure 3: Uncoated HIM micrographs of Escherichia coli when they attach onto the dragonfly wing. Images were recorded from top side. a) At lower magnification surrounding nanopillars appear to be bending towards the bacterium. Inset shows similar SEM micrograph. (Scale bar $2 \mu \mathrm{m})$. This observation is consistent with literature. b-f) At higher magnifications the 
surrounding nanopillars appeared as bent toward bacterium can be resolved to be structural component (EPS, pilus like structures) of bacterium, but not nanopillars. Scale bar $100 \mathrm{~nm}$. e) 33 degrees tilted image clearly shows that EPS are secreted onto nanopillars of dragonfly wing.

When a bacterium makes initial contact with dragonfly wing, an adhesive force is developed between the EPS and nanopillar interface. These nanoscale adhesive forces are capable of membrane deformation. ${ }^{61-63}$ Due to strong adhesion, the bacterium experiences small nanoscale membrane deformation, ${ }^{46,64}$ despite the higher stiffness of PG layer. Therefore, the bacterium experiences interfacial conditions different from that in free floating planktonic state once attached. If these conditions are not favourable, attached bacterium may attempt to move away from the surface. This leads to apply stress on the bacterial membrane. A tilted HIM Micrograph shows that bacterium is attached onto tall nanopillars at two polar ends of the bacterium and attached nanopillars are leaned towards the right (Figure S2). This suggests that the attached bacterium pushes and pulls the nanopillars while attempting to move away from the unfavourable nanopillar topography, imposing shear force onto the bacterial membrane. We have conducted the experiment using the Staphylococcus aureus bacterium. Bending of nanopillars under the bacterium, secretion of EPS and the EPS left on nanopillars were observed.

Different stages of Escherichia coli bacterial death on nanopillars can be seen in the HIM micrograph in Figure 4. These four different bacteria are at different stages of their membrane damage, hence they appear slightly different to each other. Bacterium labelled as no.1 is at its initial stage of death, just after attachment on the nanopillars. The bacterium has its rod-shaped morphology, but its membrane is slightly deformed and its cytoplasm is beginning to leak. The membrane of bacterium no.2 is wrinkled and most of its cytoplasm is leaked. At the next stage, bacterium no. 3 has lost its rod-like shape and flattened as the majority of its cytoplasm is lost 
(see arrow head). Membrane of this bacterium is still resting on the nanopillars. In the final stage the integrity is lost as shown in bacterium no.4. The height of the bacterium is similar to the nanopillars, and its cytoplasm has completely leaked out and it has sunk into the nanopillars. The leaked cytoplasm on the wing can be identified by dark colour which floods around the nanopillars. The result from this micrograph shows that a bacterium sink into nanopillars only after the membrane damage, which is in good agreement with previously reported data. ${ }^{7-9}$ As bacteria were allowed to naturally attach to the uncoated surface, they can be approached to the surface at different time intervals. Therefore, different stages of bacterial death on the wing can be identified as seen in Figure 4. However, as in Figure 3 clear visualization of secreted peripheral EPS cannot be obtained here for two reasons. Firstly, the image was obtained to show bacterial attachment onto the dragonfly wing with a larger FOV (Field of view) and the minute features close to the bacteria cannot be resolved. Secondly, as the cytoplasm is leaked out from the bacterium, EPS and cytoplasm are mixed and it is difficult to identify them separately. 


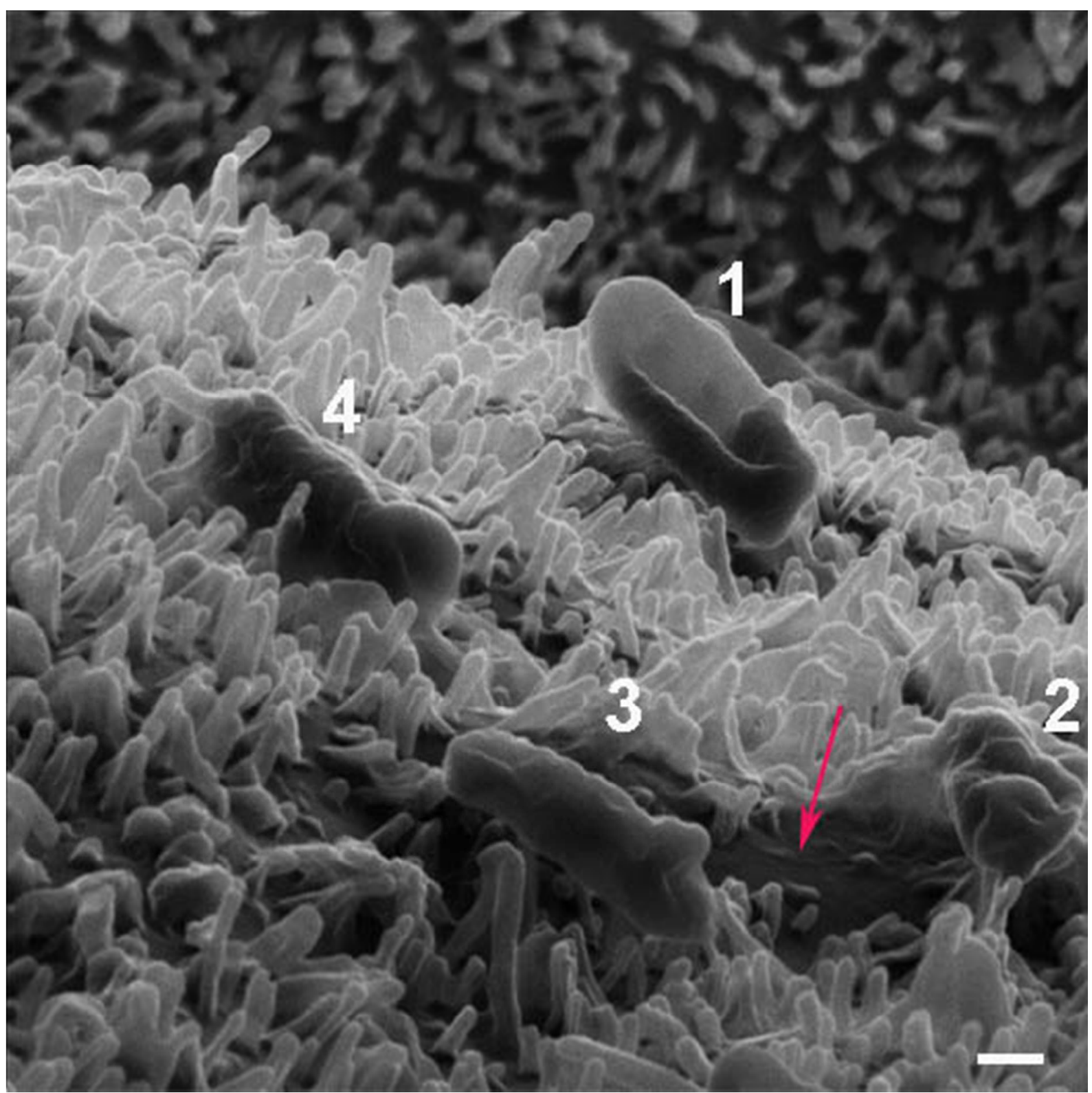

Figure 4: Four different Escherichia coli bacteria attached on uncoated nanopillar surface of dragonfly wing indicate progressive dying stages. Image was acquired without conductive metal coating in HIM. Bacterium no.1 has started leaking out its cytosol and membrane is deformed and bent inward. Bacterium still rest on nanopillars. One half of bacterium appears in grey as it has already lost the cytosol while the other half is dark as cytosol is still remaining inside. Leaked cytosol can be seen in darker color flooding nanopillars under the bacterium. Bacterium no.2 has a wrinkled outer membranes and cytosol leaked around it. Leaked cytosol is marked by 
the arrow. Bacterium labelled as no.3 has also leaked its cytosol, flattened in shape and membrane is deformed. From side it shows that bacterium is still resting on top of nanopillars. Bacterium labelled as no.4 has lost its membrane integrity and sank in to nanopillars. Leaked cytosol has flooded the nanopillars. Original images are rotated $90^{\circ}$ for best representation. Scale bar $200 \mathrm{~nm}$

PeakForce QNM ${ }^{\circledR}$ (Quantitative Nanoscale Mechanical Characterization) AFM measurements were performed to study the bacterial death in more depth. These measurements were done on uncoated sample and the measurements were done on live sample. No fixation was done before the measurements. Therefore, this measurement represents the actual events take place during bacteria-nanopillar interaction. The AFM micrograph in Figure 5 shows forming blebs, membrane irregularities/ deformations and EPS secretion in addition to cytoplasm leak on the wing. The nano-mechanical properties of the bacterium, the EPS, the leaked cytoplasm and the wing were measured. The mechanical properties were selected along the Z-Z' line (covering all interested features) and presented as graphs in Figure 5b-d to compare their variations along the topography. Different components found along the Z-Z' line is labelled and marked by double headed arrows. From $Z$ to $Z$ ' they are wing (Z-\#), EPS, Bacterium (\# to \#\#), leaked cytoplasm (\#\# to $* *)$ and the rest of the wing (** to Z'). The area for EPS is marked as a purple band and area for cytoplasm is marked as a green band in all graphs. The log modulus graph (Figure 5c) shows a larger modulus $(\log 7.5)$ for the bacteria compared to the nanopillars $(\log 7.4)$. However the area where EPS is present on bacterium and cytoplasm leak, it shows a decreased modulus. Where the bacterial cytoplasm leak and EPS secretions are present, adhesion force increases (Figure 5d). This suggests that the EPS secretions and leaked cytoplasm are sticky. The dotted line connects the topography and mechanical properties of a bleb. Measurements around the bleb 
on the top side of the bacterium shows a decreased modulus and an increased adhesion, suggesting leakage and secretion from the top side of the bacterium. If topography alone is considered, bacterium could be identified by the height, however, EPS secretions and leaked cytoplasm cannot be distinguished as the wing is a wavy structure. But with the characteristic mechanical properties, different components could be identified. When the bacteria are attached on the control glass surface, cytoplasm leak cannot be noticed. 
a

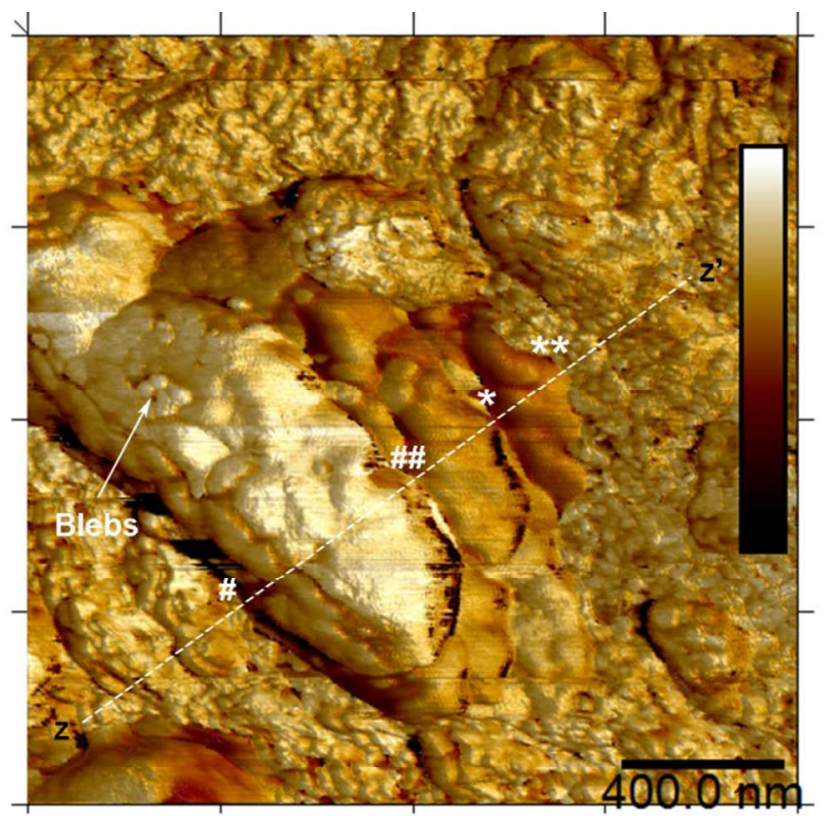

Leaked
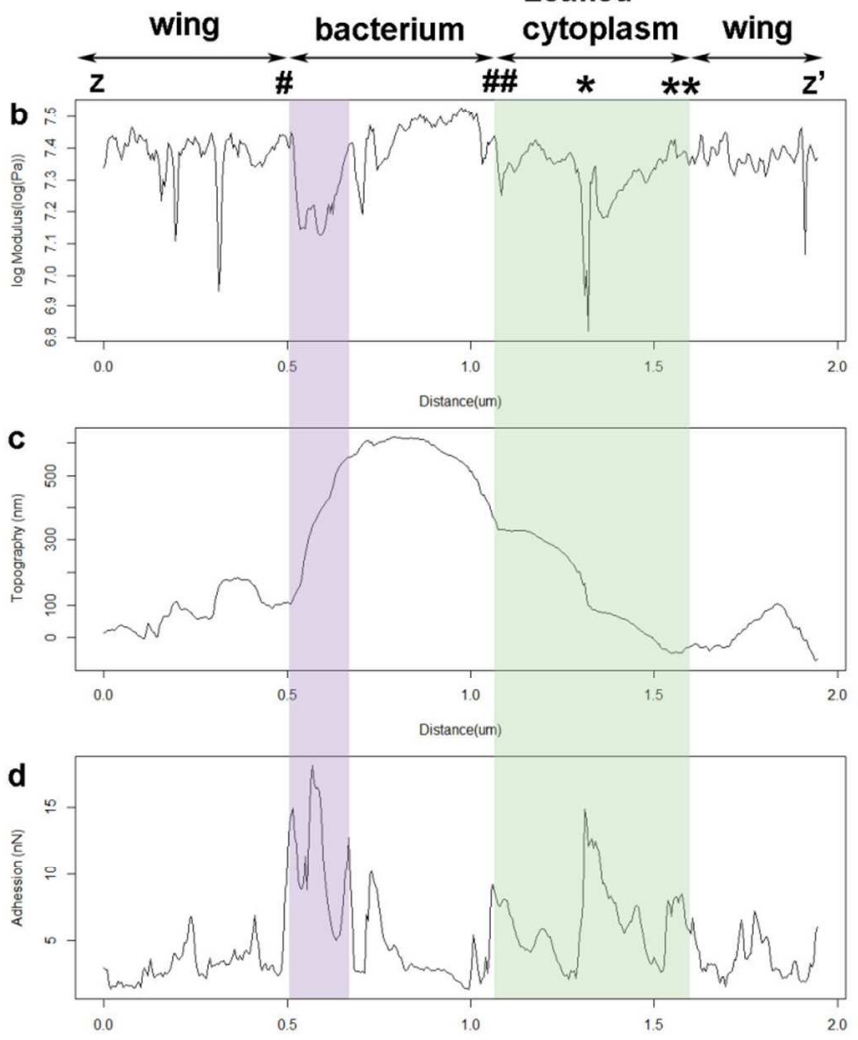

Figure 5: AFM micrograph showing a dying bacterium on a dragonfly wing with leaked cytoplasm and comparison of mechanical properties of different components on the surface. a) Log DMT modulus map of the surface. Morphological changes of the bacterium (blebs, 
irregularities) and leaked cytoplasm can also be identified. Along the z-z' line; the wing can be seen between $\mathrm{z}$ and \# as well as between ${ }^{* *}$ and $\mathrm{z}$, the bacterium is located on the line between \# and \#\#, cytoplasm leak is found from \#\# to **. b) Variation of modulus along the line z-z' in a across different components. c) Variation of topography across line z-z' marked in a. d) Adhesion force of different components along the line $z-z^{\prime}$ marked in a. Purple colored area highlights and relates the different mechanical properties and topography of EPS, while area highlighted in green relates the mechanical properties and topography of leaked cytoplasm. When EPS and cytoplasm is present on surface, adhesion is increased while modulus is decreased.

Blebs are indication of local damages to PG layer. It is possible that turgor pressure to cause the inner-membrane to push outwards against the damaged PG layer which results in forming blebs. Damage to PG layer may eventually progress to cell membrane rupture and cytoplasm leakage. The presence of such membrane irregularities on the top side of bacterium in both AFM and HIM micrographs suggests that disruption of the protective PG layer could propagate all around the cell envelope. This suggests cytoplasm leaks or secretions may be possible from the top side of the bacterium. Therefore, membrane damage not necessarily occurs at the bottom side after interaction. Similar changes of membrane morphology were observed in dead Escherichia coli exposed to high mechanical stresses, ${ }^{65}$ which suggests that bacteria on dragonfly wing may experience similar stress before they die.

To gain more insight into the membrane damage, further investigations of the bacteriumnanopillars interface were performed using Transmission Electron Microscopy (TEM) and FIB/SEM 3D tomography. The subsequent staining of the bacteria allowed visualisation of the membrane as well as the cytosol in cross-sectional TEM micrographs (Figure 6). The TEM 
cross-sectional view in Figure 6a reveals that there is no direct contact between the bacteria membrane and the nanopillars and the nano space may be filled by secreted EPS during attachment (bacterial membrane-EPS-nanopillars). Tall nanopillars are bent under the bacterium, while short nanopillars appear further below the bacterium. At this stage bacterial membrane is intact and no puncture by the nanopillars was observed although bending of the interacting taller nanopillars can be seen at far ends of bacteria as in Figure 6a. The induced strong van der Waals forces between bacterial membrane and nanopillars result the membrane to be stretched when the immobilised bacterium attempt to move on the wing surface. Previous studies showed that cylindrical shaped nanopillars are capable of applying larger shear force on a cell membrane but with a lower indentation (penetration) compared to a cone or pyramidal shaped nanopillars. ${ }^{48}$ At this early stage of attachment, the bacterium retains its rod shaped morphology, but membrane puncture was not observed. Non-puncture is consistent with the membrane rupture model ${ }^{28}$ as the modulus of the tall nanopillars are weaker than Escherichia coli PG layer (see Table 1). 


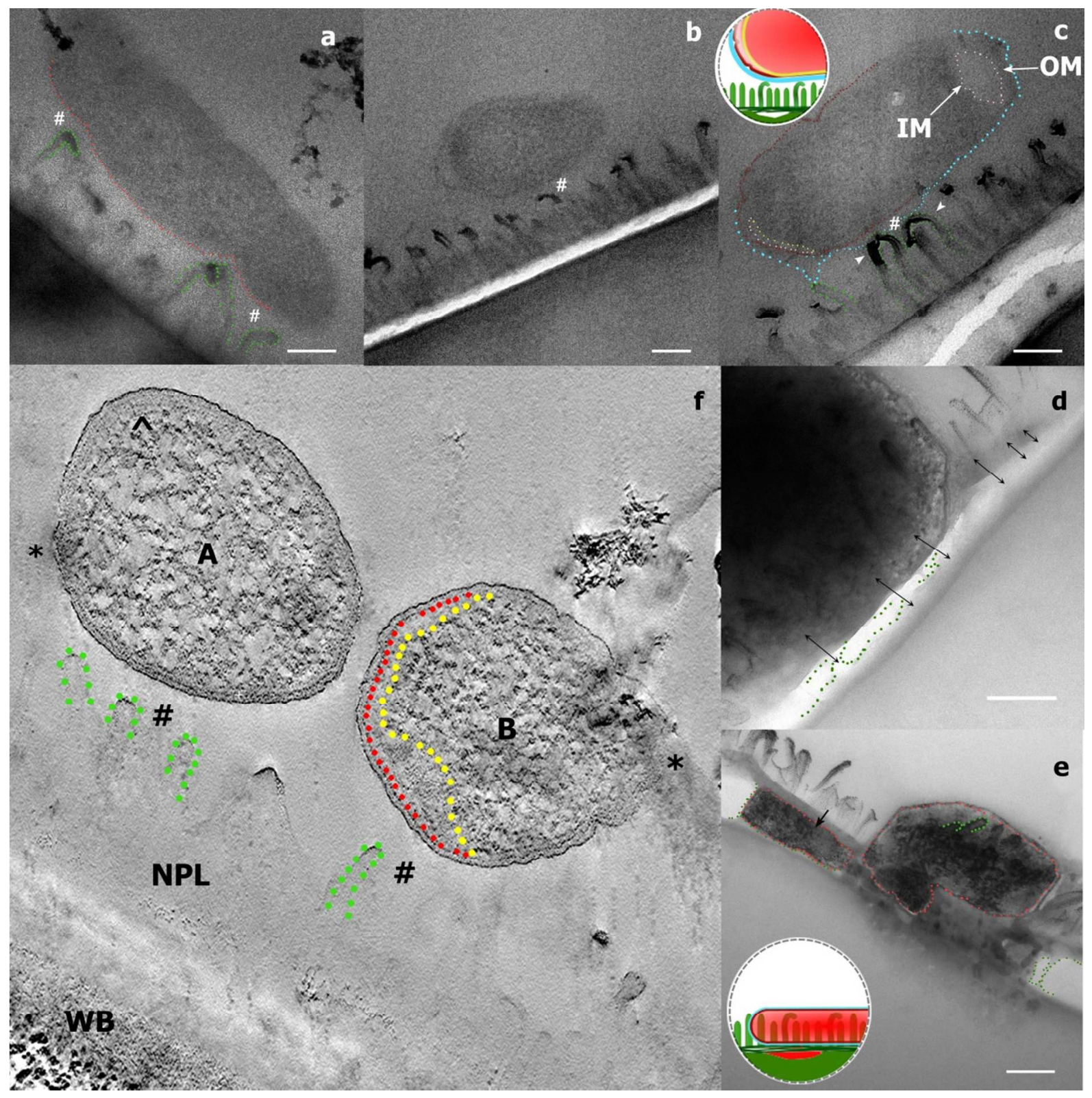

Figure 6: TEM micrographs showing bacteria-nanopillar interaction at the interface. a) Longitudinal section of E.coli bacterium approached on two tall nanopillars of a dragonfly wing topography. The membrane is still intact, and is pressed inwards. A nano scale space between bacterial membrane and nanopillars can be noted (\#). This pseudo-nano space is filled by EPS, therefore bacterium is physically attached to the nanopillars via EPS layer. As EPS do not have sufficient contrast to the surrounding under TEM, we do not see EPS as a separate layer in the image. Bending of the nanopillars underneath the bacterium is highlighted by the green dashed 
lines b) Longitudinal cross-section showing a separation of the inner membrane (IM) and outer membrane $(\mathrm{OM})$ at the polar ends of the E.coli bacterium. The tall nanopillars are bent underneath the bacterium. c) Longitudinal cross-section of a bacterium on the dragonfly wing. Bent tall nanopillars are highlighted by white arrow heads. Small gap between nanopillars and the bacterial membrane is still observed (space between red and blue lines, \#). Increased membrane separation at the polar end of the bacterium, is present. d) Nanopillar layer separation from wing base has started to form under nanopillars (marked by dotted lines). Nanopillars inside the bacterium can be noted. Double headed black arrows indicate the separation of nanopillar layer from the base of the wing. The tearing is a result by the attempts by bacterium to move away. e) The bacterium has lost its globular shape and volume to a greater extent and has shrunk into nanopillar topography. Cytoplasm is leaked under the nanopillar layer and filled the tearing under nanopillar layer (highlighted by the red dashed line) formed in $d$. f) TEM tomogram of the interface. Nanopillars are outlined with green dotted line. Membrane separation is outlined with red and yellow dotted lines. Nanopillars have a gap between membrane and they are not in contact. Dotted lines are guide to eye only. Original images are rotated $90^{\circ}$ for best representation. All scale bars correspond to $200 \mathrm{~nm}$.

Bacterial membrane damage can be identified as separation of inner-membrane (IM) and outer-membrane (OM) from each other at the polar end of the bacterium (Figure 6c). This finding is in contrast to the previous reports that membrane damage (rupture) occurred between the contact points with nanopillars. ${ }^{28-30}$ We propose that this membrane damage caused due to the strong adhesion of bacteria with the nanopillar layer via EPS and fatal shear force occurs when the immobilised bacterium attempts to move away from the surface. Effects due to attempts of movements of immobilised bacterium are evident in HIM and TEM micrographs. 
When the strongly adhered bacterium attempts to move away from the surface, it pulls the attached nanopillars towards the direction of movement (Figure S2). This process induces the separation of organic nanopillar from the wing base under the bacterium, and can be identified in the TEM micrographs (Figure 6d). At this instance stretching off of nanopillar layer from wing base can be seen and marked by the double headed arrows in Figure 6d. This can lead to the separation of nanopillar layer from wing base. A similar observation of strong adhesion and nanopillar layer separation due to movement of yeast cells on dragonfly wing nanopillars was reported. ${ }^{31}$ In the literature the strong adhesion and movement caused a cracking and peeling off of the nanopillar layer from the wing base, which was then wrapped around the dead cell. ${ }^{31}$ As a bacterium is a fraction smaller than a yeast cell, a strongly attached bacterium may not capable of peeling the nanopillar layer from the wing base as reported in yeast, but layer separations can be expected due to similar behaviour. Moreover, several studies have demonstrated strong cellsubstrate adhesion when nanopillars are present on the surface and the nanopillars can be bent by adhesion mechanisms exerted during cellular adhesion. ${ }^{27,31,66-69}$ However, the cell-nanostructure adhesion mechanisms are still poorly understood. ${ }^{68-70}$

When the bacterial membrane is damaged and cytoplasm is flooded onto wing, nanopillars can be seen inside the bacterium (Figure 6d-e). At this stage, the width of the bacterium increased and this is similar to the SEM image of dead cells in Figure 6b. Nanopillars can be seen in bacterium as the PG layer loses its integrity and the bacterium lose its shape (Figure 6e). Once the bacterial membrane has damaged and cell cytoplasm is leaked and flooded onto the surrounding nanopillars (Figure 6a), filling the cavities formed (Figure 6d) during the nanopillarbacteria interaction. Similar contrast areas both in nanopillar penetrated bacterium and under the nanopillar layer are identified in TEM micrograph (Figure 6e). These are where the formed 
cavities were filled with leaked cytoplasm. Once a bacterium has completely leaked its cytoplasm, nanopillars are penetrated into the cell and are dead; its upper membrane rests on the nanopillars as reported in the literature ${ }^{9,11}$ and in Figure $2 \mathrm{~b}$. Here the dead bacterium sunk into the nanopillars. TEM tomogram (Figure 6f) shows deformed and damaged membrane with IM and OM separated as shown by the dotted lines in bacterium B. Increased cross-sectional area of periplasm can be clearly seen in the tomogram $\left(\right.$ shown by $\left.{ }^{\wedge}\right)$ of bacterium " $A$ ". The nano space filled by EPS (shown by \#) between the tip of nanopillars and bacterial membrane shows that initial membrane damage occurs without nanopillars directly contact the outer membrane. Possible damaged areas of the membranes are seen at the side walls (shown by *).

At every adhesion, bacterium experience stress and secretes EPS. Once it experience more stress during adhesion, it secretes more EPS and vice-versa. Bacterium is attached to the surface via this secreted EPS layer. As the surface area is increased due to the nanopillar topography, adhesion is strong. The effect of nanopillars on adhesion dependent bactericidal activity is evident with TEM imaging. When the nanopillars are present, bacterial membrane separation, deformations and excess EPS secretions are clearly compared to that of a bacterium attached on a flat surface (Figure S3). The bacterium is attached to the nanopillar via secreted EPS layer and this layer (nano space, \#) is enhanced due to excessive secretion of EPS, where bacterium experiences more stress by the unfavourable nanopillar surface (Figure S3a). This leads to higher van der Waals forces and strong adhesion between bacterium and nanopillars. When there are no nanopillars, nano space (\#) between nanopillars and bacterial membrane are minimal as excess EPS secretions are not observed (Figure S3b). This comparison of the nano space between membrane and nanopillars filled by EPS indicates that a bacterium attaching on nanopillars experience more stress than a bacterium on a relatively flat surface. 
One can argue that the gap appearing could be a local artefact during TEM sample preparation due to difference in stiffness of bacteria and the wing. In the experiments the cross-sections were made in the direction perpendicular to the interface of bacteria and wing. Therefore, during the slicing, process, wing and bacterium is cut simultaneously, therefore possibility of artefact is minimal. (See supplementary figure S3, where prominent gap is noted once nanopillars are present but, gap is not significant when nanopillars are absent) However, any ambiguity in TEM preparation could potentially avoided by prepare samples at a temperature close to liquid nitrogen, which requires more specialized equipment.

To further verify that this gap between nanopillars and outer membrane at interface is not a local artefact during TEM processing, a 3D reconstruction (slice and view) of a bacterium on the dragonfly wing was performed using a FIB/SEM. The 3D tomograph shown in Figure 7 indicates that the bacterium is attached to the surface by the EPS and that the outer membrane is not in contact with the nanopillars. The inner membrane collapses and the cell leakage occurs onto the nanopillars. The obtained data is in good agreement with the TEM results. The reconstruction shows that the cytoplasm leakage (which floods the surrounding nanopillars), EPS layer and separation of the two membranes (IM and OM). The gap seen in TEM is the EPS layer secreted onto the nanopillars as confirmed by 3D tomogram. Despite the Au coating of the wing for TEM studies, the TEM results are found to be similar to the AFM, HIM and FIB/SEM observations obtained from the uncoated wing. This shows that Au coating on the wing prior to the introduction of bacteria have no effect on the bactericidal mechanism. It is to be noted that for the AFM imaging, the samples were not fixed before the imaging and for all the other techniques, samples were fixed and processed as detailed in the methodology section. Despite 
the fixation adapted in this study, AFM and all the techniques used have captured the similar effects discussed above during the bacterial death on the natural nanotopography.

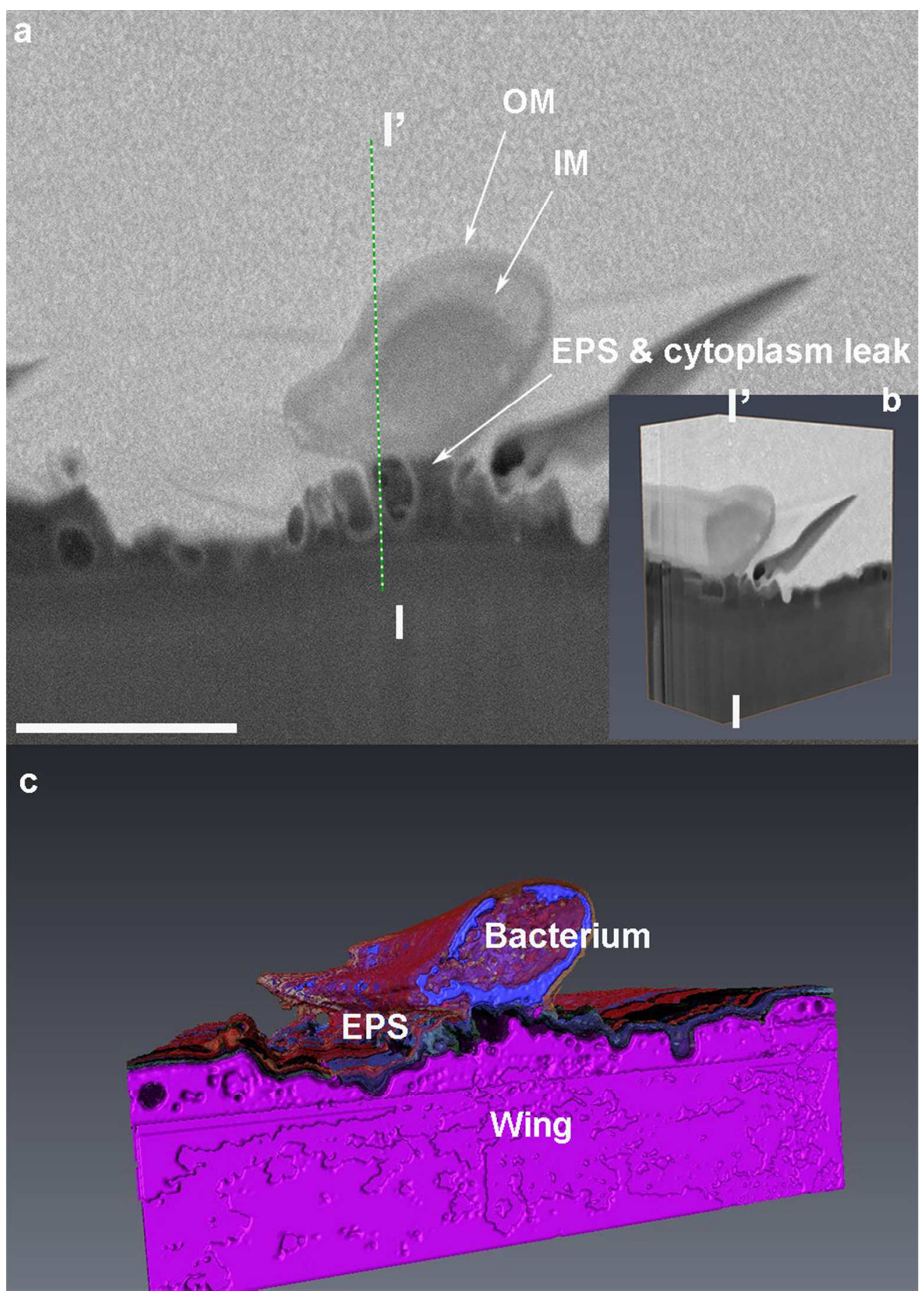

Figure 7: 3D reconstruction of a bacterium dying on the dragonfly wing a) Cross-section of the 3D reconstruction showing the bacterium - nanopillar interaction. This shows the attachment of 
bacterium to the wing surface via EPS. This indicates that outer membrane is not in contact with nanopillars. Separation of inner-membrane and outer membrane are also observed. Scale bar corresponds to $500 \mathrm{~nm} \mathrm{b)} \mathrm{Inset} \mathrm{shows} \mathrm{the} \mathrm{aligned} \mathrm{images} \mathrm{to} \mathrm{reconstruct} \mathrm{3D} \mathrm{image} \mathrm{of} \mathrm{bacterium.}$ c) The 3D reconstruction of the interface (purple: wing, blue: bacterial membrane, gray: EPS).

This research proposes a new mechanistic explanation of bactericidal activity based on dragonfly wing nanopillar topography and compared to the currently accepted model initially developed for cicada wing (Figure 8a-d). The proposed mechanism of this research is based on experimental observations and clearly demonstrates the succussive events that occur at the interface between a bacterial cell and the nanopillars (Figure 8e-h). The results show that the nanopillars are composed of two distinct height populations (Figure 8e) that are organised as a web of "nano-teeth". The nanopillars increase the surface area of the dragonfly wing, contributing to a stronger adhesion between bacteria and the nanopillars due to high van der Waals forces. Strong adhesion forces alone are lethal to bacteria. ${ }^{46}$ As bacteria secrete EPS for adhesion and survive against many environmental, biological and mechanical stresses, excess secretion of EPS is shown by a separate layer (blue layer) in Figure 8f. In return, strong shear forces are imposed on the membrane upon the bacterium's attempts to move away from the unfavourable surface. At this stage leaning of the taller nanopillars towards the moving direction and separation of nanopillar layer from wing base is observed (Figure 8g). Stronger adhesion induces membrane deformation and shear stress when immobilised bacterium attempt to move on the surface, initiate the bacterial membrane damage, which can be observed as separation of inner membrane (IM) from the outer membrane (OM) (Figure 8g). Consequently, the membrane is significantly damaged, causing cytosol to leak out, filling the cavities developed under the wing base. Ultimately, the membrane's mechanical integrity is compromised, causing the 
bacterial remnants including the membrane sink onto the nanopillar layer (Figure 8h). This finding is in contrast to the previous reports that membrane damage (rupture) occurring at the contact points with nanopillars. ${ }^{28-30}$ Our proposed mechanism provides an experimentally supported understanding of the underlying mechanism of the bactericidal properties of naturally occurring nano-topographical structure of dragonfly wing. This knowledge provides a starting point for understanding the role of adhesion as a major bactericidal property of natural nanotopography. Knowledge of nature's underlying bactericidal mechanism by NTS is essential for successful mimicking of bacteria killing surfaces.

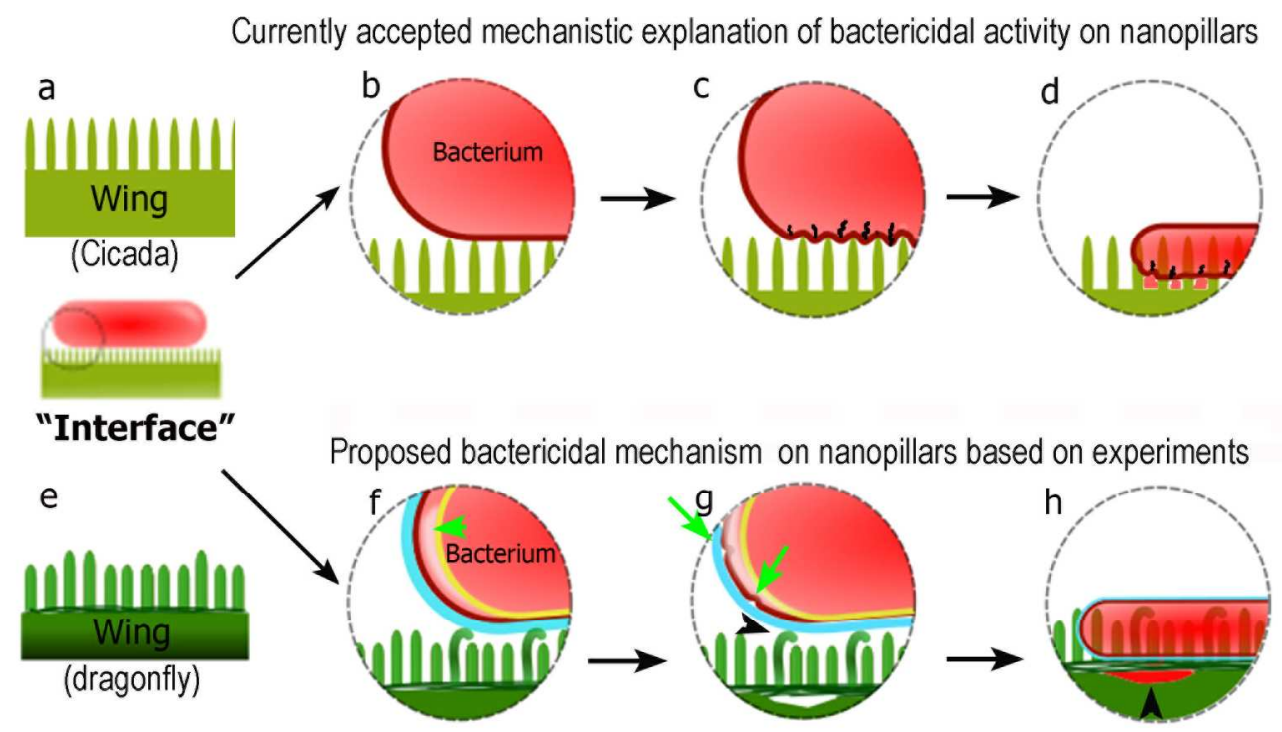

Figure 8: Proposed mechanism of bactericidal activity of nanopillars. The mechanism of bactericidal activity based on current accepted mechanistic models using cicada wing structure is shown in (a-d). The proposed mechanism based on the experimental studies in this work (using dragonfly wing) is shown in (e-h). a) Cross-section of a cicada wing was used for the current studies to determine bactericidal activity. All nanopillars are assumed to be the same in height. b) A Bacterium approaches on the surface and the membrane starts to compress due to weight and adsorbtion c) The membrane starts to rupture in between attached nanopillars due to stretching. 
The energy for stretching and membrane deformation is provided by the initial adsorbtion. d) Once cell membrane ruptures, the bacterium's cytoplasm leaks, leading to cell death on the nanopillar surface. e) Illustration of the dragonfly wing's two prominent nanopillar populations. f) Once bacteria approach to the surface, taller nanopillars are being bent by the bacterium. The nanopillars do not puncture the membrane. Bacterium adheres to the nanopillars by the secreted EPS layer and the pilus structures. Once adhesive forces apply stress on bacterial membrane, the two cell membranes of the bacterium starts to separate from each other (indicated by the arrow). The EPS layer is displayed in blue, the outer membrane in dark red and the inner membrane in yellow. g) The damage bacterial membrane starts wrinkling, and forms blebs (arrows), separation of the nanopillar layer from the wing base, also observable due to the attempts made by the bacterium to move away. h) Once the bacteria die on nanopillars, cytosol is leaked and flows under the nanopillar layer filling the crack formed in the previous step. Nanopillars can be seen inside the bacterium at this stage.

\section{CONCLUSION}

This research investigates the nanotopographical characteristics of Orthetrum villosovittatum dragonfly wing and reveals its natural bactericidal effects of Escherichia coli. Advanced microscopy techniques were utilized to study the natural surface topography of the wing and investigated the characteristics of natural interaction of bacterium and nanopillars at the interface. The results show new insights that the Orthetrum villosovittatum dragonfly wings has two-prominent height populations of cylindrical-shaped nanopillar topography and the resolved interface shows that membrane damage was initiated by a combination of strong adhesion between nanopillars and bacterium EPS as well as shear force when immobilised bacterium attempt to move away from the unfavourable surface topography. Our experimental findings are 
contrary to the commonly accepted mechanistic models (using cicada wing nanopillars) that membrane rupture was explained by direct contact of the bacterial cell membrane with the nanopillars of singular height. Our findings will help understanding the natural bactericidal effects of NTS and will allow to effectively fabricate efficient bactericidal NTS with aptitude for various disciplines and applications in future.

\section{EXPERIMENTAL SECTION}

\section{Dragonfly wing sample preparation}

Dragonflies (Orthetrum villosovittatum) were collected from the Brisbane city botanic garden area (Australia) and frozen at $-20^{\circ} \mathrm{C}$ to sacrifice the insect. Wings were then carefully dissected using a scalpel and re-attached to glass slides using double sided tape and washed with an ample amount of MiliQ water to wash any dust or particles attached. The samples were then dried at room temperature inside a laminar flow cabinet and then stored at $-20^{\circ} \mathrm{C}$ for later use.

\section{Bacterial sample preparation}

The Escherichia coli (NCTC 10418) strain was used as a model strain to study the interaction of the bacterium and the dragonfly wing at the interface. This bacterium is a typical representation of commonly available contaminant in water. Bacterial sample preparation was carried out similar to the procedure used by similar experiments ${ }^{7,9,71}$. Bacterial cultures were refreshed on nutrient agar plates from culture and were grown over night at $37^{\circ} \mathrm{C}$ in $5 \mathrm{~mL}$ of nutrient broth. The culture was collected at the logarithmic stage of growth and the bacteria suspension was washed twice with $0.01 \mathrm{M}$ PBS $(\mathrm{pH}=7.4)$ and adjusted to $\mathrm{OD}_{600}=0.3$. Aliquot of $200 \mu \mathrm{L}$ of bacterial suspension was placed on $5 \mathrm{~mm} \times 5 \mathrm{~mm}$ size wing and allowed to incubate for 30 minutes at room temperature $\left(22^{\circ} \mathrm{C}\right)$ in a Petri dish. During this procedure, bacterial cells maintain their hydrated state and allowed us to preserve the natural state of bacterial attachments 
without drying during the experiment. After the incubation period, wings were gently washed with ample amount of MiliQ water to remove non-attached cells and fixed with $2 \%$ paraformaldehyde for 20 minutes. The wings were washed again with an ample amount of MiliQ water and finally processed for imaging as required. For TEM studies, each wing was coated with a $10 \mathrm{~nm}$ Au layer prior to introduction of the bacterial culture to gain necessary contrast for microscopy.

\section{Quantitative analysis of bactericidal efficiency}

Bacterial cultures were refreshed on nutrient agar plates from a stock culture and grown over night at $37^{\circ} \mathrm{C}$ in a $5 \mathrm{~mL}$ of nutrient broth. The culture was collected at the logarithmic stage of growth and washed twice with 0.01 M PBS solution ( $\mathrm{pH}=7.4)$. An aliquot of $1 \mathrm{~mL}$ of bacterial suspension from an adjusted $\mathrm{OD}_{600}=0.1$ bacteria suspension was placed on $5 \mathrm{~mm}$ x $5 \mathrm{~mm}$ size wing and allowed to incubate for $4 \mathrm{~h}$ at room temperature $\left(22^{\circ} \mathrm{C}\right)$ in a cell culture plate. A $5 \mathrm{~mm}$ x $5 \mathrm{~mm}$ size glass piece was used for control. At every $1 \mathrm{~h}, 10 \mu \mathrm{L}$ aliquot of was taken and a 10x dilution series $\left(10^{-1}\right.$ to $\left.10^{-8}\right)$ was made and from the resulting $100 \mu \mathrm{L}$ solution, $30 \mu \mathrm{L}$ was plated on the nutrient agar media, in triplicate, for each solution. Plates were incubated over night at $37^{\circ} \mathrm{C}$, and colonies for each aliquot between 3-30 were counted and recorded with their respective dilution factor. This experiment was conducted in triplicate.

\section{Sample preparation for TEM}

\section{Membrane staining}

Bacteria samples were prepared by washing with $0.01 \mathrm{M}$ PBS and immersed in $0.1 \mathrm{M}$ cacodylate buffer twice for 10 minutes and post fixed with $1 \%$ osmium tetroxide in a cacodylate buffer for 1 hour. The samples were washed with UHQ water for 10 minutes, three times, and immersed in $1 \%$ Uranyl acetate in water for 1 hour. These samples were dehydrated with $30 \%$, 
$50 \%, 60 \%, 70 \% 90 \%$, and $3 \times 100 \%$ ethanol for 10 minutes each. Samples were resin embedded with $1: 2,1: 1,2: 1$ spurrs resin for 30 minutes, followed by $100 \%$ resin for 1 hour. Samples were embedded in fresh resin and moulded at $70 \mathrm{C}$ for 48 hours before slicing and imaging the interface under TEM.

Cytoplasm staining

Bacteria samples were prepared by firstly washing in $0.01 \mathrm{M}$ PBS (1x5 minutes bench $+2 \mathrm{x}$ $40 \mathrm{~s}$ Microwave (80 watt, vacuum on) and then inserted into $2 \%$ osmium tetroxide and $1.5 \%$ potassium ferricyanide solution for 1 hour. The samples were washed with UHQ water (1x5 minutes bench +2 x $40 \mathrm{~s}$ microwave 80 watt vacuum on), dipped in $10 \mathrm{~g} / \mathrm{L}$ thiocarbohydrazide solution for 20 minutes, then washed again with the same washing procedure The samples were then immersed in $2 \%$ osmium tetroxide for 30 minutes and washed with UHQ as in the previous washing step. The samples were next immersed in $1 \%$ uranyl acetate for 30 minutes and washed with UHQ water, as previous in the washing step. Then the samples were immersed in a lead aspartate solution $\left(0.066 \mathrm{~g}\right.$ lead nitrate $+10 \mathrm{~mL} 0.03 \mathrm{M}$ aspartic acid solution) for 1 hour at $60{ }^{\circ} \mathrm{C}$. and washed with UHQ water as in the previous step. Samples were dehydrated in $20 \%, 50 \%$, $70 \%, 90 \%, 100 \%$ and $100 \%$ ethanol solutions in the microwave for $40 \mathrm{~s}$ each, at 100 watts. LX112 resin was infiltrated by $25 \%, 50 \%$ and $75 \%$ steps on the bench for 30 minutes, followed by 3 minutes in the microwave at 150 Watts. Samples were then treated with 100\% LX112 for 30 minutes on the bench followed by 3 minutes in the microwave ( 250 watts, Vacuum). Finally, the samples were embedded in fresh resin at $70^{\circ} \mathrm{C}$ for 48 hours. Solidified samples were sliced into $100 \mathrm{~nm}$ thick slices using an ultra-microtome and placed on slot grids to be imaged under TEM. 


\section{Sample Characterization using HIM}

Biological samples are conventionally coated with conductive thin films to reduce sample charging in electron microscopy. However, visualizing the nano-topographical features of delicate biological materials such as dragonfly wings presents a challenge as coating may introduce artefacts to delicate surfaces and obscure nanoscopic features ${ }^{72}$. Helium Ion Microscope (HIM) is an ideal technique for characterizing the nanostructure of dragonfly wing without requiring a conductive coating. Charging caused by the sample's insulating properties can be compensated using a flood gun, a beam of electrons which compensate for the accumulated ion beam charge.

Fixed samples with bacteria were washed with MiliQ water and air dried. Dried wings were mounted using double sided carbon tape onto an aluminium stab for imaging. The samples were not coated as to preserve the natural nanopillar structure. High-resolution Helium Ion Microscopy images were taken by Zeiss Orion Helium Ion Microscope, at $25 \mathrm{kV}$ with a $0.3 \mathrm{pA}$ blanker current.

\section{Sample Characterization using AFM}

A $5 \mathrm{~mm}$ x $5 \mathrm{~mm}$ wing sample was mounted on a cover slip using a water drop. Once the water evaporated, a wing area of $2 \mu \mathrm{m} \times 2 \mu \mathrm{m}$ was scanned at a scan rate of $0.200 \mathrm{~Hz}$ using Bruker FASTSCAN-C tip with a spring constant of 0.4-1.2 N/m in Bruker dimension FastScan AFM with ScanAsyst. When characterizing bacteria on dragonfly wing, a fresh wing was flooded with $200 \mu \mathrm{L}$ fresh solution of bacteria in $0.01 \mathrm{M}$ PBS $\left(\mathrm{OD}_{600}=0.1 \mathrm{pH}=7.4\right)$. Surface introduced with bacterial solution was scanned using the same cantilever without fixation. Therefore, during the scan, bacteria maintain its semi-hydrated state. Results were analysed using the Nanoscope analysis $1.5 \mathrm{v}$ software which assessed the topography and mechanical properties of nanopillars. 
During the PeakForce tapping (PFT) scanning, cantilever oscillates at a frequency that is well below the cantilever resonance frequency. Force on the cantilever is measured during approach and withdraw. The tip is brought intermittently close to the sample and the deformation is in few nanometers. PFT can recognize a local maximum force at this point. This point where the tip comes off the surface is the pull-off point and the adhesion is given by the maximum force (PeakForce) at this point. Recognition of PeakForce below the base line allows the operation at very low forces; hence, this technique allows obtaining data from soft samples. ${ }^{73}$ Scan image is given by analyzing each modulation. The force curve is converted to a force vs. a separation plot, which is analogous to the load-indentation curves used in nanoindentation. ${ }^{74-75}$ Therefore, data can be extracted during a single scan using the Nanoscope software by different data acquiring channels located in the instrument.

The Young's Modulus is determined by analyzing the retract curve using the DerjaguinMuller-Toporov (DMT) model $^{57}$ in the Nanoscope analysis software;

$$
F-F_{a d h}=\frac{4}{3} E^{*} \sqrt{R\left(d-d_{0}\right)^{3}}
$$

where $F-F_{a d h}$ is the force on the cantilever relative to the adhesion force, $R$ is the tip end radius, and $d-d_{0}$ is the deformation of the sample. The result of the fit is the reduced modulus $E^{*}$. With the known Poisson's ratio, one can calculate the Young's Modulus $\left(\mathrm{E}_{\mathrm{s}}\right)$ by the equation 2 below. As the Poisson's ratio is not accurately known, we report the reduced modulus by assuming the Poisson's ratio to be 0 .

$$
E^{*}=\left[\frac{1-v_{s}^{2}}{E_{s}}+\frac{1-v_{t i p}^{2}}{E_{t i p}}\right]^{-1},
$$


where modulus of tip, $\left(\mathrm{E}_{\mathrm{tip}}\right)$ is assumed infinite compared to the soft surface.

The Energy dissipation is given by the following equation;

$$
W=\int \vec{F} \cdot d \vec{Z}=\int_{0}^{T} \vec{F} \cdot \vec{v} d t
$$

where $W$ represents energy dissipated in a cycle of interaction. Here, $F$ is the interaction force and $\mathrm{d} Z$ is the displacement. The deformation is the penetration of the tip into the surface at the peak force. This may include both elastic and plastic contributions. With the long range attraction involved, this measurement can be associated with some errors. ${ }^{74}$

\section{Sample Characterization using TEM}

A $100 \mathrm{~nm}$ thick cross-section of resin embedded dragonfly wings was imaged in the TEM. Images were analysed using ImageJ software to assess individual nanopillar height and diameter. 139 nanopillars were measured to determine the height distribution while 162 nanopillars were measured to determine the diameter distribution. The obtained data was analysed using the standard expectation-maximization algorithm for normal mixtures $\operatorname{method}^{76}$ to calculate the mean values of the two distributions using $\mathrm{R}$ statistical software, and their mean values and standard deviations were reported.

\section{The 3D reconstruction using FIB/SEM}

A $3 \mathrm{D}$ reconstruction of a bacterium on the dragonfly wing was performed using a FIB/SEM (FEI Scios) to verify the TEM measurements. TEM only allows to visualize sections of the bacteria (local information) while a FIB/SEM 3D reconstruction allows to visualize multiple sections without material loss (global information) with similar resolution. FIB/SEM does not require the sample to be embedded, artefacts caused by the resin embedding can therefore be recognized when comparing the results to TEM. 
The sample was fixed, stained and dehydrated. A single bacterium was selected using the SEM at $2 \mathrm{kV}$ acceleration voltage, $13 \mathrm{pA}$ beam current and InLens detectors. Charging issues, due to the insulating properties of the sample, required to perform a lift-out of the area of interest onto a conductive substrate. Precise cutting with the ion beam in the slice and view process was not possible otherwise. First, the area of interested was covered by depositing a $2 \mu \mathrm{m}$ thick platinum to preserve the sample surface. The region of interest (lamella) was then isolated by cutting regular cross-sections either sides with the ion beam using $30 \mathrm{kV}, 0.1 \mathrm{nA}$ ion beam current with a $20 \%$ beam overlap and $200 \mathrm{~nm}$ beam blur to avoid sample melting and heat damage. A cutting depth of $10 \mu \mathrm{m}$ and $\mathrm{Au}$ application file were chosen to achieve the required sample depth. The lamella was then lifted out and placed on a conductive TEM copper grid and covered completely with a $5 \mu \mathrm{m}$ thick secondary conductive platinum layer. The platinum helps to create a smooth surface which is required to minimize curtaining during the slice and view process and provides a conductive matrix which minimizes beam deflection due to charging.

$15 \mu \mathrm{m}$ deep trenches were then cut on either side of the tomography block using $30 \mathrm{kV} \mathrm{HV}$, $3 \mathrm{nA}$ ion beam current to prevent issues by redeposition during the slice and view process. The front face of the created tomography block was then pre-polished using $30 \mathrm{kV} \mathrm{HV}, 50 \mathrm{pA}$ ion beam current, and a cutting depth of $25 \mu \mathrm{m}$. Low ion beam currents are required to prevent sample damage. Once the area of interest (bacterium) on the dragonfly wing was reached, a final polishing process using $30 \mathrm{kV}, 10 \mathrm{pA}$ ion beam current and a depth of $25 \mu \mathrm{m}$ was performed to achieve an ultra-smooth front surface for optimal slice and view results.

The entire slice and view process of 83 slices was performed manually. The automatic Slice and View software proved not powerful enough to obtain well focussed images of the sub $100 \mathrm{~nm}$ biological features. 
$30 \mathrm{kV}$ acceleration voltage, $30 \mathrm{pA}$ ion beam current, Au application file and a cutting depth of $25 \mu \mathrm{m}$ using cleaning cross-section cuts were used for the slice and view process. The slices were recorded with the $\mathrm{T} 1$ in-lens detector (BSE) using $2 \mathrm{kV}, 6.3 \mathrm{pA}$ electron beam current, $20 \mu \mathrm{s}$ dwell time, dynamic focus and tilt correction for cross-section corrections. The acquired images were processed using FIJI and Avizo softwares. Stack alignments and contrast corrections were carried out using FIJI. All other steps were performed using Avizo 7. The smoothing: anisotropic diffusion noise reduction filter was used to improve image quality. 5 iterations and a stop threshold of 100 were used for this. The segmentation had to be performed for each slice individually using the magic wand tool. The different sample parts were distinguished by their intensity values (contrast).

\section{Associated content}

\section{Supporting Information}

The following files are available free of charge.

Figure S1: SEM images of dragonfly wing

Figure S2: HIM micrograph show Escherichia coli bacterium attempts to move on nanopillars of dragonfly wing

Figure S3: TEM tomograph compare interfaces of bacteria on nanopillar topography and a damaged area of dragonfly wing

Figure S4: Confocal micrograph of live/dead staining of Escherichia coli on dragonfly wing Figure S5: FIB/SEM data showing interface.

Table S2: Natural nanotopographies and their bactericidal efficiencies reported Table S2: Fabricated nanotopographical surfaces and their bactericidal efficiencies reported 
Section S1: Advantages and limitations of AFM and TEM approaches for measuring the height of high aspect ratio surface features.

\author{
AUTHOR INFORMATION \\ Corresponding Author \\ Dr.Tuquabo Tesfamichael \\ Science and Engineering Faculty, \\ Queensland University of Technology (QUT) \\ 02 George Street, Brisbane, QLD 4000, Australia \\ Email: t.tesfamichael@qut.edu.au \\ Tel: (+61) 0731381988 \\ Notes
}

The authors declare no competing financial interest.

\title{
Author Contributions
}

All authors contributed to this work. A.O, S.S, T.T and C.D.B, designed the research. A.O, S.S and T.T supervised the experiments. C.D.B prepared samples, characterized, analysed data and wrote initial manuscript. A.W has conducted the FIB/SEM measurements. K.O, I.A, A.O, T.T and S.S read manuscript and improved. A.O and C.D.B proposed the current mechanism. All authors discussed the results and commented on the manuscript.

\section{Acknowledgement}


This work is supported by the Queensland University of Technology. C.D.B. acknowledges the QUT Postgraduate Research Award (QUT PRA), SEF scholarship and tuition fee scholarship from the Queensland University of Technology. The authors acknowledge the facilities and the scientific and technical assistance of Dr Peter Hines, Dr Jamie Riches, Rachel Hancock, Erica Loves and Ning Liu at the Australian Microscopy \& Microanalysis Research Facility (AMMRF) at the Central Analytical Research Facility (CARF), Queensland University of Technology, Brisbane, Australia. Authors also acknowledge the facilities (FEI Scios), and the scientific and technical assistance of Dr Hui Diao, of the Australian Microscopy \& Microanalysis Research Facility at the Centre for Microscopy and Microanalysis, The University of Queensland and important discussions with Dr. Rick Webb at The University of Queensland. Authors also acknowledge the support and technical assistance by Dr. Juliana Chirutha at Faculty of Health, Queensland University of Technology (QUT), the support and facility (Bruker Dimension FastScan) by Dr. Christian Gow from Coherent scientific. Authors acknowledge the support from the colleagues Jagath Senarathne and Mahasen Dehideniya for their valuable discussions for statistical data analysis and the QUT Academic Language and Learning Service (ALLS), especially Karyn Gonano, in the writing and development of this document.

\begin{abstract}
Abbreviations
EPS extracellular polymeric substance; FOV field of view; HIM helium ion microscopy; IM inner-membrane; NPL nano-pillar layer; NTS nano textured surface; OM outer-membrane.
\end{abstract}




\section{References}

1. Lee, H.; Lee, B. P.; Messersmith, P. B., A Reversible Wet/dry Adhesive Inspired by Mussels and Geckos. Nature 2007, 448, 338-341.

2. Autumn, K.; Peattie, A. M., Mechanisms of Adhesion in Geckos. Integr. Comp. Biol. 2002, 42, 1081-1090.

3. Malshe, A.; Rajurkar, K.; Samant, A.; Hansen, H. N.; Bapat, S.; Jiang, W. P., Bioinspired Functional Surfaces for Advanced Applications. Cirp Annals-Manufacturing Technology 2013, 62, 607-628.

4. Siddique, R. H.; Gomard, G.; Holscher, H., The Role of Random Nanostructures for the Omnidirectional Anti-reflection Properties of the Glasswing Butterfly. Nat Commun 2015, 6.

5. Morikawa, J.; Ryu, M.; Seniutinas, G.; Balčytis, A.; Maximova, K.; Wang, X.; Zamengo, M.; Ivanova, E. P.; Juodkazis, S., Nanostructured Antireflective and Thermoisolative Cicada Wings. Langmuir 2016, 32, 4698-4703.

6. Dellieu, L.; Sarrazin, M.; Simonis, P.; Deparis, O.; Vigneron, J. P., A Two-in-one Superhydrophobic and Anti-reflective Nanodevice in the Grey Cicada Cicada orni (Hemiptera). J. Appl. Phys. 2014, 116, 024701.

7. Ivanova, E. P.; Hasan, J.; Webb, H. K.; Gervinskas, G.; Juodkazis, S.; Truong, V. K.; Wu, A. H.; Lamb, R. N.; Baulin, V. A.; Watson, G. S.; Watson, J. A.; Mainwaring, D. E.; Crawford, R. J., Bactericidal Activity of Black Silicon. Nat Commun 2013, 4, 2838.

8. Mainwaring, D. E.; Nguyen, S. H.; Webb, H. K.; Jakubov, T.; Tobin, M.; Lamb, R.; Wu, A. H.-F.; Marchant, R.; Crawford, R. J.; Ivanova, E. P., The Nature of Inherent Bactericidal Activity: Insights from the Nanotopology of Three Species of Dragonfly. Nanoscale 2016, 6, 6527-6534.

9. Ivanova, E. P.; Hasan, J.; Webb, H. K.; Truong, V. K.; Watson, G. S.; Watson, J. A.; Baulin, V. A.; Pogodin, S.; Wang, J. Y.; Tobin, M. J.; Löbbe, C.; Crawford, R. J., Natural Bactericidal Surfaces: Mechanical Rupture of Pseudomonas Aeruginosa Cells by Cicada Wings. Small 2012, 8, 2489-2494.

10. Kelleher, S. M.; Habimana, O.; Lawler, J.; O’ Reilly, B.; Daniels, S.; Casey, E.; Cowley, A., Cicada Wing Surface Topography: An Investigation into the Bactericidal Properties of Nanostructural Features. ACS Applied Materials \& Interfaces 2016,. 8, 14966-74

11. Hasan, J.; Webb, H. K.; Truong, V. K.; Pogodin, S.; Baulin, V. A.; Watson, G. S.; Watson, J. A.; Crawford, R. J.; Ivanova, E. P., Selective Bactericidal Activity of Nanopatterned Superhydrophobic Cicada Psaltoda Claripennis Wing Surfaces. Appl. Microbiol. Biotechnol. 2012, 97, 1-6.

12. Ensikat, H. J.; Ditsche-Kuru, P.; Neinhuis, C.; Barthlott, W., Superhydrophobicity in Perfection: the Outstanding Properties of the Lotus Leaf. Beilstein Journal of Nanotechnology 2011, 2, 152-61.

13. Liu, Y.; Li, G., A new method for producing "Lotus Effect" on a Biomimetic Shark Skin. J. Colloid Interface Sci. 2012, 388, 235-242.

14. Sun, T.; Feng, L.; Gao, X.; Jiang, L., Bioinspired Surfaces with Special Wettability. Acc. Chem. Res. 2005, 38, 644-652.

15. Byun, D.; Hong, J.; Saputra; Ko, J. H.; Lee, Y. J.; Park, H. C.; Byun, B.-K.; Lukes, J. R., Wetting Characteristics of Insect Wing Surfaces. Journal of Bionic Engineering 2009, 6, 63-70. 
16. Hensel, R.; Finn, A.; Helbig, R.; Braun, H.-G.; Neinhuis, C.; Fischer, W.-J.; Werner, C., Biologically Inspired Omniphobic Surfaces by Reverse Imprint Lithography. Adv. Mater. 2014, 26, 2029-2033.

17. Guadarrama-Cetina, J.; Mongruel, A.; Medici, M. G.; Baquero, E.; Parker, A. R.; Milimouk-Melnytchuk, I.; Gonzalez-Vinas, W.; Beysens, D., Dew Condensation on Desert Beetle Skin. Eur. Phys. J. E Soft Matter 2014, 37, 109.

18. Bhushan, B.; Jung, Y. C.; Koch, K., Self-Cleaning Efficiency of Artificial Superhydrophobic Surfaces. Langmuir 2009, 25, 3240-3248.

19. Jung, Y. C.; Bhushan, B., Wetting Behavior of Water and Oil Droplets in Three-Phase Interfaces for Hydrophobicity/philicity and Oleophobicity/philicity. Langmuir 2009, 25, 1416514173.

20. Kesel, A. B., Aerodynamic Characteristics of Dragonfly Wing Sections Compared with Technical Aerofoils. J. Exp. Biol. 2000, 203, 3125-3135.

21. Young, J.; Walker, S. M.; Bomphrey, R. J.; Taylor, G. K.; Thomas, A. L., Details of Insect Wing Design and Deformation Enhance Aerodynamic Function and Flight Efficiency. Science 2009, 325, 1549-1552.

22. Walsh, M. J., Riblets as a Viscous Drag Reduction Technique. AIAA journal 1983, 21, 485-486.

23. Ivanova, E. P.; Nguyen, S. H.; Webb, H. K.; Hasan, J.; Truong, V. K.; Lamb, R. N.; Duan, X.; Tobin, M. J.; Mahon, P. J.; Crawford, R. J., Molecular Organization of the Nanoscale Surface Structures of the Dragonfly Hemianax papuensis Wing Epicuticle. PLoS One 2013, 8, e67893.

24. Dickson, M. N.; Liang, E. I.; Rodriguez, L. A.; Vollereaux, N.; Yee, A. F., Nanopatterned Polymer Surfaces with Bactericidal Properties. Biointerphases 2015, 10, 021010.

25. Bhadra, C. M.; Khanh Truong, V.; Pham, V. T. H.; Al Kobaisi, M.; Seniutinas, G.; Wang, J. Y.; Juodkazis, S.; Crawford, R. J.; Ivanova, E. P., Antibacterial Titanium Nanopatterned Arrays Inspired by Dragonfly Wings. Sci. Rep. 2015, 5, 16817.

26. Wang, X.; Bhadra, C. M.; Yen Dang, T. H.; Buividas, R.; Wang, J.; Crawford, R. J.; Ivanova, E. P.; Juodkazis, S., A Bactericidal Microfluidic Device Constructed Using NanoTextured Black Silicon. RSC Advances 2016, 6, 26300-26306.

27. Sjöström, T.; Nobbs, A. H.; Su, B., Bactericidal Nanospike Surfaces via Thermal Oxidation of Ti Alloy Substrates. Mater. Lett. 2016, 167, 22-26.

28. Pogodin, S.; Hasan, J.; Baulin, V. A.; Webb, H. K.; Truong, V. K.; Phong Nguyen, T. H.; Boshkovikj, V.; Fluke, C. J.; Watson, G. S.; Watson, J. A.; Crawford, R. J.; Ivanova, E. P., Biophysical Model of Bacterial Cell Interactions with Nanopatterned Cicada Wing Surfaces. Biophys. J. 2013, 104, 835-840.

29. Xue, F.; Liu, J.; Guo, L.; Zhang, L.; Li, Q., Theoretical Study on the Bactericidal nature of Nanopatterned Surfaces. J. Theor. Biol. 2015, 385, 1-7.

30. Li, X., Bactericidal Mechanism of Nanopatterned Surfaces. Phys. Chem. Chem. Phys. 2016, 18, 1311-1316.

31. Nowlin, K.; Boseman, A.; Covell, A.; LaJeunesse, D., Adhesion-dependent Rupturing of Saccharomyces Cerevisiae on Biological Antimicrobial Nanostructured Surfaces. Interface 2014, 12, 20140999

32. Song, F.; Lee, K. L.; Soh, A. K.; Zhu, F.; Bai, Y. L., Experimental Studies of the Material Properties of the Forewing of Cicada (Homoptera, Cicadidae). J. Exp. Biol. 2004, 207, 3035-42. 
33. Vincent, J. F. V.; Wegst, U. G. K., Design and Mechanical Properties of Insect Cuticle. Arthropod Structure \& Development 2004, 33, 187-199.

34. Gorb, S. N., Porous channels in the Cuticle of the Head-arrester System in Dragon/Damselflies (Insecta: Odonata). Microsc. Res. Tech. 1997, 37, 583-591.

35. Kreuz, P.; Arnold, W.; Kesel, A. B., Acoustic Microscopic Analysis of the Biological Structure of Insect Wing Membranes with Emphasis on their Waxy Surface. Ann. Biomed. Eng. 2001, 29, 1054-1058.

36. Wan, Y.-1.; Cong, Q.; Wang, X.-j.; Yan, Z., The Wettability and Mechanism of Geometric Non-Smooth Structure of Dragonfly Wing Surface. Journal of Bionic Engineering 2008, 5, 40-45.

37. Li, X.; Chen, T., Enhancement and Suppression Effects of a Nanopatterned Surface on Bacterial adhesion. Physical Review E 2016, 93, 052419.

38. Berne, C.; Ducret, A.; Hardy, G. G.; Brun, Y. V., Adhesins Involved in Attachment to Abiotic Surfaces by Gram-negative Bacteria. Microbiology spectrum 2015, 3, 10.1128/microbiolspec.MB-0018-2015.

39. Flemming, H.-C.; Wingender, J., The Biofilm Matrix. Nat Rev Micro 2010, 8, 623-633.

40. Donlan, R. M., Biofilms: Microbial Life on Surfaces. Emerg. Infect. Dis. 2002, 8, 881890.

41. Sutherland, I. W., Biofilm Exopolysaccharides: a Strong and Sticky Framework. Microbiology 2001, 147, 3-9.

42. Katsikogianni, M.; Missirlis, Y. F., Concise review of mechanisms of bacterial adhesion to biomaterials and of techniques used in estimating bacteria-material interactions. European Cells and Materials 2004, 8, 37-57.

43. Suo, Z.; Avci, R.; Deliorman, M.; Yang, X.; Pascual, D. W., Bacteria Survive Multiple Puncturings of Their Cell Walls. Langmuir 2009, 25, 4588-4594.

44. Avci, R.; Suo, Z.; Deliorman, M.; Yang, X.; Pascual, D. W., Self-repair Of Bacterial Cell Wall Against Multiple Puncturings By An AFM Tip. Biophys. J. 2009, 96, 450a-451a.

45. Sahoo, S.; Rao, K. K.; Suraishkumar, G. K., Reactive Oxygen Species Induced by Shear Stress Mediate Cell Death in Bacillus Subtilis. Biotechnol. Bioeng. 2006, 94, 118-127.

46. Liu, Y.; Strauss, J.; Camesano, T. A., Adhesion Forces Between Staphylococcus Epidermidis and Surfaces Bearing Self-assembled Monolayers in the Presence of Model Proteins. Biomaterials 2008, 29, 4374-4382.

47. Tiller, J. C.; Liao, C.-J.; Lewis, K.; Klibanov, A. M., Designing Surfaces that Kill Bacteria on Contact. Proceedings of the National Academy of Sciences 2001, 98, 5981-5985.

48. Obataya, I.; Nakamura, C.; Han, S.; Nakamura, N.; Miyake, J., Mechanical Sensing of the Penetration of Various Nanoneedles into a Living Cell Using Atomic Force Microscopy. Biosens. Bioelectron. 2005, 20, 1652-1655.

49. Galtung, J., Theory and Methods of Social Research Allen \& Unwin: 1968.

50. Weber, N. A., Dimorphism in the African Oecophylla Worker Nd an Anomaly (Hym.: Formicidae). Ann. Entomol. Soc. Am. 1946, 39, 7-10.

51. Eyre-Walker, A.; Keightley, P. D., The Distribution of Fitness Effects of New Mutations. Nat Rev Genet 2007, 8, 610-618.

52. Deng, Y.; Sun, M.; Shaevitz, J. W., Direct Measurement of Cell Wall Stress Stiffening and Turgor Pressure in Live Bacterial Cells. Phys. Rev. Lett. 2011, 107, 158101. 
53. Dokukin, Maxim E.; Guz, Nataliia V.; Sokolov, I., Quantitative Study of the Elastic Modulus of Loosely Attached Cells in AFM Indentation Experiments. Biophys. J. 2013, 104, 2123-2131.

54. Tuson, H. H.; Auer, G. K.; Renner, L. D.; Hasebe, M.; Tropini, C.; Salick, M.; Crone, W. C.; Gopinathan, A.; Huang, K. C.; Weibel, D. B., Measuring the Stiffness of Bacterial Cells from Growth Rates in Hydrogels of Tunable Elasticity. Mol. Microbiol. 2012, 84, 874-891.

55. Yao, X.; Jericho, M.; Pink, D.; Beveridge, T., Thickness and Elasticity of Gram-negative Murein Sacculi Measured by Atomic Force Microscopy. J. Bacteriol. 1999, 181, 6865-6875.

56. Minne, S.; Hu, Y.; Hu, S.; Pittenger, B.; Su, C., NanoScale Quantitative Mechanical Property Mapping Using Peak Force Tapping Atomic Force Microscopy. Microsc. Microanal. 2010, $16,464-465$.

57. Maugis, D., Contact, Adhesion and Rupture of Elastic Solids. Springer Berlin Heidelberg: 2000.

58. Xiao, K.; Bai, K.; Wang, W.; Song, F., Experimental Study on the Microstructure and Nanomechanical Properties of the Wing Membrane of Dragonfly. Acta Mechanica Sinica 2007, 23, 281-285.

59. Smith, C. W.; Herbert, R.; Wootton, R. J.; Evans, K. E., The Hind Wing of the Desert Locust (Schistocerca Gregaria Forskal). II. Mechanical Properties and Functioning of the Membrane. J. Exp. Biol. 2000, 203, 2933.

60. Gosline, J. M., The Elastic Properties of Rubber-like Proteins and Highly Extensible Tissues. Symp. Soc. Exp. Biol. 1980, 34, 332-357.

61. Tiller, J. C., Antimicrobial Surfaces. In Bioactive Surfaces, Börner, G. H.; Lutz, J.-F., Eds. Springer Berlin Heidelberg: Berlin, Heidelberg, 2011, 193-217.

62. Schaer, T. P.; Stewart, S.; Hsu, B. B.; Klibanov, A. M., Hydrophobic polycationic Coatings that Inhibit Biofilms and Support Bone Healing During Infection. Biomaterials 2012, 33, 1245-1254.

63. Chen, Y.; Harapanahalli, A. K.; Busscher, H. J.; Norde, W.; van der Mei, H. C., Nanoscale Cell Wall Deformation Impacts Long-Range Bacterial Adhesion Forces on Surfaces. Appl. Environ. Microbiol. 2014, 80, 637-643.

64. Busscher, H. J.; van der Mei, H. C., How Do Bacteria Know they are on a Surface and Regulate their Response to an Adhering State? PLoS Pathog. 2012, 8, e1002440.

65. Arakha, M.; Saleem, M.; Mallick, B. C.; Jha, S., The Effects of Interfacial Potential on Antimicrobial Propensity of ZnO Nanoparticle. Sci. Rep. 2015, 5, 9578.

66. Parsons, J. T.; Horwitz, A. R.; Schwartz, M. A., Cell Adhesion: Integrating Cytoskeletal Dynamics and Cellular Tension. Nature reviews. Molecular cell biology 2010, 11, 633-643.

67. Ahn, J.; Son, S. J.; Min, J., The Control of Cell Adhesion on a PMMA Polymer Surface Consisting of Nanopillar Arrays. J. Biotechnol. 2013, 164, 543-548.

68. Kuo, C. W.; Chueh, D.-Y.; Chen, P., Investigation of Size-dependent Cell Adhesion on Nanostructured Interfaces. Journal of Nanobiotechnology 2014, 12, 54.

69. Sjöström, T.; McNamara, L. E.; Meek, R. M. D.; Dalby, M. J.; Su, B., 2D and 3D Nanopatterning of Titanium for Enhancing Osteoinduction of Stem Cells at Implant Surfaces. Advanced Healthcare Materials 2013, 2, 1285-1293.

70. Hsiao, Y.-S.; Luo, S.-C.; Hou, S.; Zhu, B.; Sekine, J.; Kuo, C.-W.; Chueh, D.-Y.; Yu, H.h.; Tseng, H.-R.; Chen, P., 3D Bioelectronic Interface: Capturing Circulating Tumor Cells onto Conducting Polymer-Based Micro/Nanorod Arrays with Chemical and Topographical Control. Small 2014, 10, 3012-3017. 
71. Truong, V. K.; Lapovok, R.; Estrin, Y.; Rundell, S.; Wang, J. Y.; Fluke, C. J.; Barnes, D. G.; Crawford, R. J.; Ivanova, E. P., The Influence of Nano-scale Surface Roughness on Bacterial Adhesion to Ultrafine-grained Titanium. Biomaterials 2010, 31, 3674.

72. Joens, M. S.; Huynh, C.; Kasuboski, J. M.; Ferranti, D.; Sigal, Y. J.; Zeitvogel, F.; Obst, M.; Burkhardt, C. J.; Curran, K. P.; Chalasani, S. H.; Stern, L. A.; Goetze, B.; Fitzpatrick, J. A. J., Helium Ion Microscopy (HIM) For the Imaging of Biological Samples at Sub-nanometer Resolution. Sci. Rep. 2013, 3, 3514.

73. Kaemmer, S. B., Introduction to Bruker's ScanAsyst and PeakForce Tapping AFM Technology Application Note \#133.

74. Pittenger, B.; Erina, N.; Su, C. Quantitative Mechanical Property Mapping at the Nanoscale with PeakForce QNM, Bruker Corporation Application Note AN128 2012. http://www.bruker.com/fileadmin/user_upload/8-PDFDocs/SurfaceAnalysis/AFM/ApplicationNotes/AN128-RevB0-

Quantitative_Mechanical_Property_Mapping_at_the_Nanoscale_with_PeakForceQNMAppNote.pdf (accessed 06/11/2015).

75. Pettinger, B.; Erina, N.; Su, C., Quantitative Mechanical Property Mapping at the Nanoscale with Peak Force QNM. Bruker Appl. Note 2010, 128, 1-12.

76. Meng, X.-L.; Rubin, D. B., Maximum Likelihood Estimation via the ECM Algorithm: A General Framework. Biometrika 1993, 80, 267-278. 
Table of Content figure

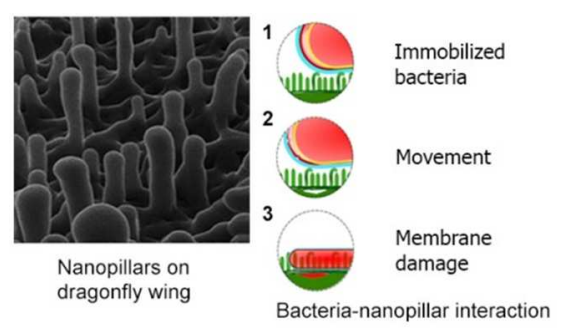

